\title{
Der Riot als Vorbote und Symptom einer Krise: Zu den Ausschreitungen während des G20-Gipfels 2017 in Hamburg
}

\begin{abstract}
Sarah Uhlmann*
Zusammenfassung

Während des G2o-Gipfels in Hamburg kam es zu immensen Ausschreitungen, die zahlreiche Diskussionen in der Presse nach sich zogen. In diesen stand häufig die Straffälligkeit der RandaliererInnen im Vordergrund. Eine weitergehende politische oder wissenschaftliche Einordnung der Geschehnisse suchte man vergebens. Dieses Defizit ist auch dem Umstand geschuldet, dass die hiesige Soziale Bewegungs- und Protestforschung kaum Forschungsansätze zur Analyse von Ausschreitungen anbietet. Der vorliegende Artikel versteht Riots als ein gesellschaftliches Phänomen und bemüht sich daher um ein tiefergehendes Verständnis, indem sowohl ideelle und psychosoziale als auch sozialstrukturelle Ursachen beleuchtet werden. Die Ausschreitungen werden mithilfe von politökonomischen Arbeiten wie die von Joshua Clover als ein Krisenphänomen gedeutet, wobei sich die Eruption selbst nur aus einer relationalen Perspektive begreifen lässt. Das Ausmaß in Hamburg war nur möglich, weil die heterogenen Protestgruppierungen beim Anblick der geteilten Gegnerschaft zur Polizei eine Koalition bildeten, in der die üblichen Grenzen zwischen den unterschiedlichen politischen Spektren aber auch sozialen Schichten kurzzeitig aufgehoben wurden und so eine schwer kontrollierbare Protestmasse entstand.
\end{abstract}

Schlagwörter: G20, Hamburg, Ausschreitungen, Protestbewegung, politische Ökonomie

\section{The riot as harbinger of crisis: On the excesses during the G20 summit 2017 in Hamburg}

\section{Abstract}

During the G2o Summit in Hamburg we have seen immense riots, which led to numerous discussions in the press. In these, the delinquency of the rioters was often in the foreground. A more far-reaching political or scientific interpretation of the events was clearly missing. This deficit is also a result of the fact that social movement and protest studies hardly offer any research approaches for the analysis of riots. The present article understands riots as a social phenomenon and therefore strives for a deeper understanding by shedding light on ideological and psychosocial as well as socio-structural causes. The riots will be interpreted as a crisis phenomenon with the help of political-economic works like those by Joshua Clover, but the eruption itself can only be understood from a relational perspective. The scale of the riots in Hamburg was only possible because the heterogeneous protest groups formed a coalition in view of their shared opposition to the police, in which the usual boundaries between the different political spectra but also social strata were briefly overturned, thus creating a mass of protestors that was difficult to control.

Keywords: G20, Hamburg, riots, protest movements, political economy

\footnotetext{
*Sarah Uhlmann, Berlin Graduate School of Social Sciences, Humboldt-Universität zu Berlin, E-Mail: sarah.uhlmann@ hu-berlin.de

Ich möchte mich bei Janna Frenzel, Philippe Greif und Fabian Klein bedanken. Der Text wäre ohne die vorangegangenen Diskussionen im Arbeitskreis, Riot' des Instituts für Protest- und Bewegungsforschung nicht zustande gekommen.
} 


\section{Einleitung}

Im Juli 2017 trafen sich die führenden RegierungsvertreterInnen zum G20-Gipfel in Hamburg. Während des Gipfels kam es neben zahlreichen Demonstrationen und Blockadeversuchen zu Ausschreitungen, immensen Sachbeschädigungen und Plünderungen, sodass manche BeobachterInnen von einer neuen Dimension der Gewalt sprachen.

Insbesondere der Freitagabend, der auf Zeit Online als die "Schlacht auf der Schanze“ (Polke-Majewski 8. Juli 2017) einging, war lange Gegenstand öffentlicher Debatten: Nachdem die Polizei die Protestierenden in Richtung Schulterblatt abgedrängt hatte, errichteten mehrere Hundert Militante zahlreiche Barrikaden und warfen Schaufenster ein. Unzählige Menschen schlossen sich an und eine Zerstörungswut machte sich breit. Die Polizei hatte sich zwischenzeitlich zurückgezogen und überließ das Viertel weitestgehend den randalierenden, mittlerweile über 1500 Jugendlichen (Eder/ Kasumov 8. Juli 2017). Geschäfte wurden geplündert und Brände gelegt. Gegen Mitternacht beendete die Polizei die Ausschreitungen mit dem Sondereinsatzkommando, das mit gezogener Schnellfeuerwaffe das Schulterblatt und umliegende Straßen räumte.

PolitikerInnen aller Couleur und auch VertreterInnen der Linkspartei, die Teile der G20-Proteste in Hamburg unterstützte, verurteilten im Nachhinein die Ausschreitungen als kriminelle und zuweilen sogar terroristische Straftaten. Selbst ein Vertreter der Roten Flora, einem seit fast dreißig Jahren besetzten autonomen Zentrum in Hamburg, delegitimierte die Riots als „sinnentleerte Gewalt“ (NDR 11. Juli 2017). Weitergehende Erklärungen für die Ausschreitungen suchte man in der Presse vergebens.

In Anbetracht des Ausmaßes der Randale während des G20-Gipfels und der anschließenden öffentlichen Debatte sollten wir jedoch, statt einer entpolitisierten Deutung der Ereignisse zu folgen, nach den gesellschaftlichen Gründen fragen. Wenn zahlreiche junge Menschen ohne Rücksicht auf mögliche Gefahren für sich und mitunter auch für andere an Riots teilnehmen und auch das Risiko eingehen, strafrechtlich belangt zu werden, können die Ursachen für solche Ereignisse, wie schon Arbeiten zu den Ausschreitungen in London 2011 gezeigt haben (Dzudzek/Müller 2013; Reicher/ Stott 2011; Slater 2011), nicht auf kriminelles oder moralisch defizitäres Verhalten Einzelner reduziert werden. Vielmehr müssen solche gewaltvollen Eruptionen in Beziehung zur Gesellschaft gesehen werden.

\section{Riots in der Protestforschung}

Auch aus sozialwissenschaftlicher Perspektive beschäftigte man sich bisher kaum mit den Gründen für die Ausschreitungen während des G20-Gipfels, was einer unzulänglichen Auseinandersetzung durch die Protestforschung mit Riots im Allgemeinen geschuldet ist. Obwohl durch ein Kollektiv ausgeübte gewaltvolle Protestformen durchaus im Spektrum politischer Artikulation verortet werden (Tilly 2003), sind sie immer noch weitestgehend unterforscht (Seferiades/ Johnston 2012a: 4; Della Porta 2006: 2). Es besteht nicht einmal ein wissenschaftlicher Konsens über die Begriffsbestimmung eines Riots (Frenzel u. a. 2016: 11f. Della Porta/Gbikpi 2012: 87). Insbesondere die Soziale Bewegungsforschung, die in Deutschland die Protestforschung maßgeblich geprägt hat, kommt bei der Analyse von Ausschreitungen an ihre Grenzen, da sie primär organisierten Protest untersucht, der sozialen Wandel verhindern oder herbeiführen möchte. Riots, wie wir sie in Hamburg gesehen haben, fallen aufgrund ihres Charakters aus dem Analyseraster. Sie sind von kurzer Dauer und scheinen auf keine politische Gestaltung abzuzielen.

Gegenstand der meisten wissenschaftlichen Arbeiten zu Riots bilden die Ausschreitungen, wie sie sich in den armen Vororten oder Vierteln von London (2011), Paris (v.a. 2005) und Ferguson (2014) ereignet haben, die eine gewisse Analogie aufweisen: Infolge einer Kontrolle durch die Polizei, bei der rassifizierte ${ }^{1}$ Jugendliche als potenzieller Gefährder erschossen wurden oder auf der Flucht vor der Polizei zu Tode gekommen sind, entzündeten sich stunden- und zuweilen tagelange Riots. Ähnlich wie Étienne Balibar und Ben Trott argumentieren auch andere AutorInnen, die die Mischung aus sozioökonomischer Ungleichheit und Rassismus, oder anders ausgedrückt „the double exclusion of class and race" (Balibar 2007: 57), im Zusammenhang mit dem konfliktiven Verhältnis zwischen Polizei und marginalisierten Jugendlichen als Grund für die Ausschreitungen ansehen (Trott 2014; Waddington/King 2012; Slater 2011; Balibar 2007).

Schon auf den ersten Blick wird klar, dass sich die in den armen Vororten und marginalisierten Stadtteilen ereigneten Riots deutlich von jenen unterscheiden,

1 Dieser Begriff geht auf das englische „racialized“ zurück und wird im Folgenden verwendet um People of color zu bezeichnen, wobei zugleich der Konstruktionsprozess rassistischer Zugehörigkeiten deutlich werden soll. 
wie wir sie in Hamburg während des G20-Gipfels oder auch regelmäßig am 1. Mai in Berlin gesehen haben. Während es sich bei ersteren um Ausschreitungen von sozial benachteiligten und meist rassifizierten Jugendlichen handelt, die infolge eines als Polizeigewalt wahrgenommenen Ereignisses ausbrechen, sind die Ausschreitungen in Deutschland häufig von anarchistischen sowie anderen linksradikalen Kreisen getragen und zuweilen auch geplant, wobei es sich hier eher um Angehörige der weißen Mittelschicht handelt (Thompson 2010; Seferiades/Johnston 2012b; Martinez 2004). Jedoch lassen sich die jeweiligen Ereignisse als Riots kategorisieren, da sie, wie folgende Begriffsbestimmung lautet, „Unruhen größeren Ausmaßes“ darstellen, „die mit der Zerstörung von Eigentum und gewalttätigen Auseinandersetzungen mit der Polizei einhergehen" (Jary und Jary zit. nach Rucht 2016: 25). ${ }^{2}$ Ohne die hier exemplarisch auf zwei Formen des Riots zugespitzten Situationen gleichsetzen zu wollen, lässt sich zum einen fragen, inwiefern die benannten Analysen zu Riots von marginalisierten Jugendlichen auch auf Ausschreitungen während des G2O-Gipfels bezogen werden können. Zum anderen gilt es der von Seraphim Seferiades und Hank Johnston aufgeworfenen Frage nachzugehen, inwiefern diese Form des gewaltvollen Protestes die Art und Weise ist, in der sich „contentious politics“ in Krisenzeiten ausdrückt (Seferiades/Johnston 2012a: 3).

Im Folgenden befasse ich mich daher mit den sozioökonomischen und politischen Ursachen, wie sie im Nachgang der Ausschreitungen durch Jugendliche in marginalisierten Vierteln herausgearbeitet wurden. Dabei stütze ich mich vor allem auf Joshua Clover, der nicht nur die ökonomische Exklusion als Grund anführt, sondern mithilfe der politischen Ökonomie die derzeitige Konjunktur der Riots in den frühindustrialisierten Ländern zu erklären vermag. Auch wenn eine materialistische, sprich strukturelle Analyse, unabdingbar ist, kann eine Deutung der Ausschreitungen nicht ohne eine Auseinandersetzung mit der Handlungsperspektive erfolgen. Daher wird im Anschluss auch die affektive und ermächtigende Dimension für die Teilnehmenden von kollektiven Protesthandlungen beleuchtet und auf Riots bezogen. Anschließend wird überprüft, inwiefern diese Begründungszusammenhänge auf die Ausschreitungen während des G2o-Gipfels anwendbar sind. Im vorliegenden Artikel orientiere ich mich dabei an den Untersuchungskonzepten sozi-

2 Über die Unklarheit des Riot-Begriffs in der sozialwissenschaftlichen Forschung siehe Frenzel u.a. (2016, 11ff.). aler Missstände, fehlender Möglichkeitsstrukturen, geteilter Identität und Ermächtigung, die Steve Reicher und Cliff Stott als zentrale Begründungszusammenhänge für Riots bestimmt haben (Reicher/Stott 2011: 77). Die hierbei aufgeworfenen materiellen, ideellen und sozialen Ursachen bilden zwar eine notwendige, jedoch keine hinreichende Erklärung für die Eskalation der Gewalt. Die Ausschreitungen selbst lassen sich im Anschluss an Charles Tilly lediglich relational begreifen: Der konkrete Ausbruch eines Riots lässt sich nur mit einer Analyse der „interpersonalen Prozesse“ (Tilly 2003: 20), sprich der „Transaktionen zwischen Personen und Gruppen“ (Tilly 2003: 5), erklären.

Im Folgenden untersuche ich die Riots von Hamburg als ein soziales Phänomen. Ich stelle dabei die Ausschreitungen von Hamburg als ein Symptom einer gesellschaftlichen Krise dar und begreife die Riots zugleich als das Resultat einer polarisierenden Dynamik, in der eine schwer zu kontrollierende Protestmasse entstand, die jedoch keinem kollektiven politischen Subjekt entsprach oder mit einem solchen gleichzusetzen wäre. Der vorliegende Artikel kann keine Chronologie der Protesttage ${ }^{3}$ darstellen oder einen Überblick über die ProtestakteurInnen geben, sondern untersucht vielmehr den Riot als politisches Artikulationsmittel. Zwar ist der Begriff Riot, wie Tilly zu Recht bemerkt, kein analytischer, da er aus der Alltagssprache kommt und häufig mit einer politischen Verurteilung einhergehe (Tilly 2003: 18). Doch auch wenn die politischen Konnotationen, sowohl in abgrenzender als auch befürwortender Hinsicht, kaum zu negieren sind, soll der folgende Artikel einen Beitrag

3 Trotz alledem soll hier ein kurzer Überblick geboten werden (ausführlicher siehe Frenzel/Knopp, Philipp/ Ullrich 2018). Die Woche Anfang Juli begann mit zähen Auseinandersetzungen um die Errichtung der Zelte für die AktivistInnen. Am Mittwoch wurde der von über 70 Gruppen und Nichtregierungsorganisationen ausgerichtete Gegengipfel eröffnet, in der Innenstadt fand die Kunstaktion 1000 Gestalten und am Abend fand die Nachtanzdemo Lieber Tanz ich als G2O mit 20.000 Menschen statt. Donnerstagabend wurde die autonome und antikapitalistische Demonstration aufgrund von Vermummung der TeilnehmerInnen von der Polizei angegriffen und aufgelöst, bevor diese starten konnte. Am Freitag, den Beginn des Gipfels, versuchten Tausende von AktivistInnen durch Blockaden den Beginn der Veranstaltung zu behindern. Außerdem wurde der Hafen partiell bestreikt. Am Abend ereigneten sich die immensen Ausschreitungen im Schanzenviertel, die im Zentrum dieses Artikels stehen. Zur großen Abschlussdemonstration unter dem Motto „Globale Solidarität statt G20“ fanden sich am Samstag über 70.000 Menschen ein. 
dazu leisten, eine soziologische Perspektive auf Riots bzw. Ausschreitungen zu stärken, indem diese als eine Form auf dem Kontinuum der kollektiven politischen Artikulationsweise verstanden werden. ${ }^{4}$

Die empirische Untermauerung der Thesen bleibt - dem Gegenstand geschuldet - notgedrungen unvollkommen. Riots sind einer sozialwissenschaftlichen Analyse mithin nur schwer zugänglich, da die AkteurInnen, ihr sozialer und politischer Hintergrund, ihre Organisationszusammenhänge, ihre politischen Motivationen als auch Forderungen meist im Verborgenen bleiben. Auch quantitative Erhebungen, die die Wahrnehmung der sich in den vergangenen Jahren häufenden Ausschreitungen bestätigen und wiederlegen, existieren nach meiner Kenntnis nicht. Um die Dynamik der Gipfelproteste, die Beweggründe von Riotern, den Vorgang der Ausschreitungen am Freitagabend rekonstruieren zu können, wurden daher Artikel zu den Ausschreitungen sowohl aus der Tagespresse als auch aus Publikationen der linken Bewegung qualitativ ausgewertet sowie diverse Gespräche mit Anwesenden geführt. Auch war ich während des Gipfels in Hamburg, wobei ich die Ausschreitungen im Schanzenviertel nicht erlebt, sondern nur über den Livestream von Russia Today verfolgt habe. Hierin zeigt sich zugleich die Ambivalenz eines politischen Forschungsgegenstandes, der mit einer persönlichen Involviertheit, die ich lieber aufdecken, statt verschweigen möchte, einhergeht. Wäre ich nicht aus politischer Überzeugung zu den Protesten gefahren, wäre mein Zugang zum Forschungsfeld deutlich begrenzter.

\section{3. Über den Zusammenhang von Ökonomie und kollektiven Protestformationen}

Diverse AutorInnen nennen die steigende soziale Ungleichheit als Ursache für die Riots in England (2011), Frankreich (v.a. 2005) und den USA (2014) (Trott 2014; Pritchard/Pakes 2014; Dzudzek/Müller 2013; Reicher/ Stott 2011; Slater 2011). Obwohl der durchschnittliche Wohlstand, sprich das Bruttosozialprodukt, steigt, lässt sich eine anwachsende Marginalisierung von Bevölkerungsgruppen beobachten, die sich auch räumlich

4 Aufgrund des soziologischen Zugriffs auf das Thema Riots, bei dem es primär darum geht, die gesellschaftlichen Ursachen und Hintergründe $\mathrm{zu}$ verstehen, ist die Gesetzeswidrigkeit einzelner Protestaktionen nicht Gegenstand des Artikels. niederschlägt (Wacquant 2006: 23). Die hohe Jugendarbeitslosigkeit in den von Riots betroffenen Gebieten ist ein offensichtlicher Beleg dieser These. ${ }^{5}$ Auch Befragungen von Jugendlichen, die an den Riots 2011 in England teilnahmen, anwesend oder mit Riotern befreundet waren, haben die fehlende Zukunftsperspektive, die polizeilichen Kontrollmaßnahmen und die soziale Ungleichheit als Gründe für den Ausbruch der kollektiven Gewalt ermittelt. Demnach waren die Ausschreitungen und Plünderungen „a response to repeated stop-and-search, racist policing, deprivation, poverty, unemployment, cuts to the educational maintenance allowance (EMA), anger, and inequalities between the haves and the have-nots" (James 2011). Ähnlich wie Trott, der die neoliberale Umstrukturierung als makroökonomische Ursachen für die Ausschreitungen analysiert (Trott 2014), sehen sowohl Balibar als auch Loïc Wacquant den Grund für die zunehmenden Riots in den Transformationen der Arbeitswelt. So bedeuten Arbeitsverträge nicht mehr automatisch einen Schutz vor Armut und darüber hinaus würden Teile der Bevölkerung ökonomisch überflüssig werden (Balibar 2007: 58; Wacquant 2006: 25f.).

Eine weitergehende materialistische Deutung der politischen und sozioökonomischen Kontextbedingungen für Ausschreitungen, die mit sozialen Missständen und mangelnden Partizipationsmöglichkeiten einhergehen, bietet Clover an. In seinem Buch Riot. Strike. Riot (Clover 2016) untersucht er anhand von Kategorien der politischen Ökonomie den Zusammenhang zwischen sozialen Konfliktformationen und der makroökonomischen Transformation des Kapitalismus, indem er die Entwicklung der sozialen Kämpfe der letzten Jahrhunderte in den frühindustrialisierten Ländern betrachtet. Während des 17. und 18. Jahrhunderts rebellierten die Aufständigen demnach gegen hohe Nahrungspreise, sie randalierten, plünderten auf den Marktplätzen oder blockierten die Ausfuhr von Lebensmitteln. Die präindustriellen Riots fanden somit am Ort des Tauschs und, wie Clover folgert, in der Zirkulationssphäre statt. Mit der Ausbreitung des industriellen Kapitalismus verlagerte sich der primäre soziale Konflikt in die Fabriken und dadurch in den Arbeitsbereich und in die Sphäre der kapitalistischen

5 Demnach beträgt die Jugendarbeitslosigkeit in den französischen Vororten 40 Prozent und auch in der Metropolregion von St. Louis (und somit auch Ferguson) sind 47 Prozent der afroamerikanischen Jugendlichen ohne Arbeit (Thrasher 19. August 2014; Bareis/Bojadzijev 2011: 846). 
Produktion. Kämpften die Aufständigen in der von Riots geprägten präindustriellen Ära also um die Preise der Güter, so wird der Kampf um Reproduktion im Zeitalter der Streiks über den Preis der Arbeitskraft, sprich den Lohn, ausgetragen (Clover 2016: 15). Auch Arbeitskämpfe konnten in dieser Zeit durchaus gewaltvoll sein; so wurden im Kampf um bessere Löhne oft Produktionsmaschinen und Waren zerstört, wie etwa das historische Beispiel der Maschinenstürmer aufzeigt. Die sozialstaatlichen Maßnahmen und die Institutionalisierung der Interessensgegensätze entschärften im Laufe der Zeit die soziale Frage und befriedeten und zivilisierten den zugrundeliegenden sozialen Konflikt.

Doch dieses Integrationssystem gerät zunehmend in Gefahr. Der Grund hierfür liegt im Wandel des Kapitalismus, wie Clover mit Rückgriff auf Robert Brenner, Fernand Braudel und Giovanni Arrighi zu zeigen vermag. Das Jahr 1973 mit der Ölpreiskrise stellt dabei die entscheidende Wende dar. Durch die Einbußen im industriellen Sektor sahen sich die Unternehmen gezwungen, nach neuen Märkten zu suchen. Kapital floss nicht nur in Produktionsstätten im Globalen Süden, sondern auch zunehmend in Finanzinstrumente und Handelsunternehmen, da hier höhere Profitraten warteten (Clover 2016: 132). ${ }^{6}$ Vor dem Hintergrund der seit den 1970er-Jahren einsetzenden Deindustrialisierung in den sogenannten westlichen Ländern, aufgrund derer Teile der Bevölkerung für den Arbeitsmarkt überflüssig wurden, verlieren immer mehr Menschen den Glauben an einen ökonomischen Aufstieg und an die politische Gleichheit.

Betrachtet man die sozialen Kämpfe in einer historischen Längsperspektive, wird deutlich, dass in deren Zentrum die menschliche Reproduktion steht. Der Ort dieser Kämpfe, so Clover, ist dabei an die dominante Form der Kapitalakkumulation geknüpft: Mit dem schleichenden Bedeutungsrückgang der (industriellen) Lohnarbeit verliert der Arbeitsplatz als Verhandlungsort sozialer Konflikte an Bedeutung. Daher finden im frühindustrialisierten Westen soziale Konflikte immer weniger in den Fabriken, sondern zunehmend auf den Straßen und Plätzen statt, die, wie Clover es beschreibt, Orte der Zirkulation, „der Zirkulation von Waren und Gütern“ sind (Clover 2016: 173f.). Im Vergleich zu den

6 Wie außerdem David Harvey herausarbeitet, bildet der Immobilienmarkt eine entscheidende Anlage für überschüssiges Kapital, was wiederum deutliche Konsequenzen für die Stadtentwicklung und den Wohnungsmarkt nach sich zieht (Harvey 2012). eingehegten Arbeitskämpfen in Unternehmen, die aufgrund des fehlenden Wirtschaftswachstums zunehmend defensiver sind, verlaufen die Ausschreitungen unkontrollierter und gewaltvoller. Somit sind Riots, ähnlich wie die Streiks, ein Kampf um die Reproduktion - jedoch in ihrer Negation, denn sie stellen keinen Weg dar, mit dem die Anwesenden ihren Anteil am Gewinn erhöhen könnten (Clover 2016: 29). Es ist das Protestmittel der „Überflüssigen“, um es mit Clover zuzuspitzen: „riot is the modality through which surplus is lived" (Clover 2016: 170).

Wie Clover zugleich betont, darf die Rückkehr der Riots seit den 1970er-Jahren jedoch nicht als reine Wiederholung gedeutet werden, da Neuerungen in der Wertschöpfung und im politischen System auf die kollektiven Protestformationen Auswirkungen haben. Während sich die präindustriellen Riots hauptsächlich am Hafen oder auf Marktplätzen ereigneten, finden die heutigen Randale auf den Straßen und Plätzen statt. Der Grund für diesen Unterschied sieht er dem Umstand geschuldet, dass damals „der Staat fern und die Ökonomie nah" war (Clover 2016: 29). Güter und Lebensmittel wurden lokal hergestellt und eine Polizei oder staatliche Kontrolle, wie wir sie heute kennen, gab es nicht. Heute lässt sich die Wirtschaft aufgrund der globalen Arbeitsteilung und Ausdifferenzierung kaum noch konkret fassen, wohingegen die Polizei aufgrund der Durchdringung des Staates allerorts präsent ist. Demnach zeichnen sich die heutigen Randale im Gegensatz zu den Riots im 17. und 18. Jahrhundert, die auf die ökonomische Sphäre zielten, vor allem durch eine Konfrontation mit der staatlichen Ordnungsmacht, der Polizei, aus (Clover 2016: 171ff.).

Einen weiteren Unterschied heutiger Riots im frühindustrialisierten Westen erkennt Clover in der Rassifizierung der Ausschreitungen (Clover 2016: 160). Clover macht uns darauf aufmerksam, dass sowohl die Riots von Marginalisierten als auch die Rassifizierung im Allgemeinen nicht außerhalb ökonomischer Strukturen begriffen werden können. Er bezieht sich auf Stuart Hall, der betont, wie sich für Schwarze die Klasse als race relation vermittelt: Race „ist also die Modalität, in der Klasse ,gelebt' wird, das Medium, in dem Klassenverhältnisse erfahren werden, die Form, in der sie angeeignet und ,durchkämpft" werden“" (Hall 1994: 133). Die Industrialisierung bewegte unzählige nichtweiße Menschen durch Arbeitsangebot zum Umzug und inkludierte sie durch Lohnarbeitsverhältnisse zumindest teilweise in die kapitalistische Produktion seien es die GastarbeiterInnen in Deutschland oder die 
Schwarzen, die aus dem Süden der USA in den Norden umzogen. Gleichsam sind es bevorzugt MigrantInnen und rassifizierte Menschen, die bei einsetzender Rezession entlassen werden, weshalb sie überproportional von Arbeitslosigkeit betroffen sind (Clover 2016: 161).

Da in Nordamerika und Europa für die kommende Zeit keine grundlegenden Änderungen hinsichtlich der aktuellen Produktions- und Akkumulationsbedingungen $\mathrm{zu}$ erwarten sind oder, anders ausgedrückt, kein nennenswertes Wirtschaftswachstum zu erwarten ist, sieht Clover für die nächsten fünf bis vierzig Jahre eine Zeit kommen, die verstärkt von Ausschreitungen, Blockaden, Barrikaden, Plünderungen und Besetzungen geprägt sein wird (Clover 2016: 29, 175). Ihm zufolge sind wir in eine neue Epoche von Riots getreten.

\section{Affekte und Ermächtigung im Riot}

Neben den sozialen Missständen und fehlenden Möglichkeitsstrukturen sind für ein Verständnis von Riots, folgen wir Scott und Reicher (Reicher/Stott 2011: 77), auch die geteilte Identität und Fragen der Ermächtigung entscheidend, wobei ich die daran geknüpften affektiven Anteile in den Vordergrund rücke. Wie bewegungssoziologische Arbeiten gezeigt haben, sind Gefühle notwendiger Bestandteil jeglichen kollektiven Handelns, und die Fortführung einer kollektiven Aktion ist umso wahrscheinlicher, je mehr emotionale Energie und Aufregung produziert werden (Aminzade/McAdam 2001: 14; Jasper 2011: 296). Lange Zeit wurde der Riot von Seiten der kollektiven Verhaltenstheorien als ein emotionaler und irrationaler Ausbruch von wütenden Menschenmassen angesehen. Im Gegensatz $\mathrm{zu}$ den früheren massenpsychologischen Ansätzen betonen jedoch zeitgenössische AutorInnen, dass man die Gleichsetzung von Emotionen und Irrationalität aufgeben solle (Jasper 2011: 285), da Gefühle behilflich sind, Situationen zu bewerten und Probleme oder Gefahren zu erkennen (Seferiades/Johnston 2012a: 13; Goodwin/ Jasper/Polletta 2004: 416). Auch lässt sich fragen, ob es überhaupt eine von Gefühlen getrennte Rationalität gibt. Signifikant ist zudem, dass Gefühle nicht auf einen individuellen Ursprung reduziert werden können, denn sie werden, so Craig Calhoun, ebenso durch kulturelle Verständnisse und soziale Interaktionen bedingt (Calhoun 2001: 47). So können Gefühle der Feindseligkeit, des Hasses und der Missgunst durch das Erleben von sozialer Ungleichheit und durch die negative Erfahrung mit der Polizei geschürt werden (Seferiades/Johnston 2012a: 12, Tiratelli 2018: 78). Ebenfalls werden, wie wis- senschaftliche Arbeiten zeigen, durch Ausschlüsse vom Arbeitsmarkt negative Emotionen produziert. So sind es vor allem männliche Jugendliche, die durch „strukturelle Diskriminierung auf dem Arbeitsmarkt, in deren Folge sie einem geschlechtsspezifischen patriarchalen Erwartungsdruck im Rahmen gesellschaftlich legitimer bzw. akzeptierter Modi nicht entsprechen können, in ihrem Selbstwert schwerwiegend beeinträchtigt" werden (Greif 2012: 16).

Diese negativen Gefühle, deren Entstehung im Vorfeld solcher Ausschreitungen verortet liegen, müssen in Beziehung zu jenen Emotionen gesetzt werden, die durch die Teilnahme an den Unruhen produziert werden. Wie Tiratelli anhand von Videomaterial der London Riots belegt, suche während des Riots nur ein kleiner Teil eine wirkliche direkte physische Konfrontation mit der Polizei, vielmehr entstehe das „emotionale Hoch“ dadurch, dass man durch die Riots wenigstens für kurze Zeit die Orte „besitze“, an denen man alltäglich verkehrt, an denen man zu Hause ist und zu denen man eine emotionale Bindung hat, die aber ansonsten durch die Polizei kontrolliert würden (Tiratelli 2018: 77). So sei der Riot für diejenigen eine berauschende Erfahrung, die sich „im Alltag dominiert und misshandelt fühlen“, da sie im Riot die eigenen Regeln setzen und kurzzeitig Macht demonstrieren können (Reicher/Stott 2011: 47).

Doch das Machtgefühl ergibt sich nicht allein aus der Raumaneignung, sondern fußt auf einer kurzweiligen Kollektivität. Wie aus einer geteilten Problemwahrnehmung, sprich der gefühlten Zugehörigkeit zu einer diskriminierten Gruppe, ein Gefühl von „Empowerment" erwachsen kann, erklären Reicher und Stott. Damit ein Riot entstehe, bedürfe es eines Gefühls der Einheit, das meist durch den auslösenden Vorfall der wahrgenommenen Polizeigewalt generiert werde. Somit wird auch deutlich, dass die evozierten Emotionen nicht ohne eine Identifikation mit einer Gruppe denkbar sind. Die meist männlichen Jugendlichen haben das Gefühl, „im gemeinsamen Boot zu sitzen“, den gleichen Missständen und Ausschlussmechanismen zu unterliegen und Wut oder Verachtung zu teilen (Reicher/Stott 2011: 40-43). „All this creates the confidence that, if any one person were to act, others would join in and support them" (Reicher/Stott 2011: 40). Auch laut Randall Collins, der sich auf einer mikro-soziologischen Ebene mit Gewalt auseinandergesetzt hat, produziere eine kollektive Gewalthandlung, wie sie der Riot darstelle, emotionale Energie und Solidarität unter den Teilnehmenden (Collins 2008: 9, 19). Es ist diese Art von kollektiver Ermächtigung, die sich im Riot zeigt, denn 
rioten kann man nicht allein, dafür braucht es ein Kollektiv (Reicher/Stott 2011: 41, 43).

\section{Sozioökonomischer Kontext von Hamburg}

Die benannten Arbeiten zu den Riots in den armen Stadtteilen und Vororten von London und Paris sehen die Gewaltausbrüche vor allem als eine Folge sozioökonomischer Bedingungen, die dazu führen, dass Teile der Gesellschaft ökonomisch nicht mehr gebraucht werden würden. Die Ausschreitungen seien daher ein Ausdruck einer „überschüssigen Bevölkerung“ (Clover 2016: 155), sie stellten eine „bewusste Absage an eine Mehrheitsgesellschaft und an ein politisches System“ dar, „Von dem sich viele Aufständische nichts mehr erhoffen“ (Altenried: 53). Darüber hinaus ist ein im Alltag verankertes, konfliktives Verhältnis zwischen Polizei und Jugendlichen charakteristisch, wobei den Ordnungskräften immer wieder rassistische Handlungen vorgeworfen werden. Um zu überprüfen, inwiefern die vorangestellten Aussagen auch auf hiesige Verhältnisse zutreffend sind, sollen im Folgenden die sozioökonomischen Entwicklungen in Hamburg und Deutschland beleuchtet werden.

Auch für Deutschland lässt sich für die 1970er-Jahre eine wirtschaftspolitische Zäsur erkennen (Dörre 2012: 51), deren Folgen eine nähere Betrachtung verlangen. ${ }^{7}$ Das fordistische Produktionssystem geriet in die Krise, denn die „Individualisierung von Lebensstilen und Kundenwünschen sorgte für Kollision mit einem auf standardisierte Massenproduktion ausgerichteten Produktionssystem “ (Dörre 2012: 51). Der Fordismus der Nachkriegszeit, der sich durch eine den Großteil der Erwerbstätigen integrierende und dadurch soziale Konflikte vermittelnde Lohnarbeitsgesellschaft auszeichnete (Dörre 2012: 47), ${ }^{8}$ wurde durch einen finanzialisierten Kapitalismus abgelöst, ${ }^{9}$ der

7 Bis zur Ölkrise und der damit zusammenhängenden Rezession waren in Deutschland Arbeitslosenzahlen unter 2 Prozent üblich. Dann kletterte die Arbeitslosenzahl in den 1980er-Jahren auf über 8 Prozent an und fiel und stieg konjunkturbedingt - in den kommenden Jahren. Denn niedrigsten Wert seit Anfang der 1970iger wurde 2017 mit 3,8\% Prozent erreicht, wobei angemerkt werden muss, dass Arbeit heutzutage nicht mehr gleichbedeutend mit sozialer Absicherung ist.

8 Gerne wird das Bild eines sozialintegrativen Fordismus gezeichnet. Frauen waren nur partiell Teil der Lohnarbeitsgesellschaft und bekamen, wenn sie arbeiteten, ähnlich wie ArbeitsmigrantInnen, deutlich weniger Gehalt.

9 Die Finanzmärkte stellen von den 1990ern bis zur globalen Krise 2008 den Wirtschaftszweig mit dem stärksten Wachstum dar (Dörre 2012: 55). mit atypischen und prekären Beschäftigungsverhältnissen einhergeht (Dörre 2012: 65; Castel 2008: 349). ${ }^{10}$ Doch nicht nur für Geringqualifizierte erhöhte sich die soziale Unsicherheit. Weder Mittelschichtszugehörigkeit noch akademischer Bildungsgrad schützen heute vor unsicheren Arbeits- und Lebensverhältnissen (Dörre 2015: 68). Der derzeitige Kapitalismus lebt, wie Dörre zuspitzt, „Von der Angst vor dem sozialen Abstieg“ (Dörre 2012: 78). Zudem lohnt sich ein geschlechtsspezifischer Blick auf diese Entwicklung: Immer mehr Frauen wollen ökonomisch unabhängig sein oder sind finanziell darauf angewiesen, einer Arbeit, die überwiegend in Teilzeit, zu prekären Bedingungen und in der Dienstleistungsbranche ausgeführt wird, nachzugehen. Während also der Anteil der arbeitenden Frauen sukzessive steigt, sinkt der Anteil der arbeitenden männlichen Bevölkerung. ${ }^{11}$ Seit 2005 sind erstmalig relativ gesehen mehr Männer als Frauen erwerbslos (BpB 2010).

Doch die Herausforderungen bestehen für das Individuum nicht allein im Arbeitsleben: Sie betreffen grundsätzlich die menschliche Reproduktion. Während diese früher durch die „Trias von Erwerbarbeit, Sozialstaat und Familie" (Jürgens 2012: 276) sichergestellt, d.h. „stark kollektiv reguliert und institutionell eingebettet“ war, entwickelt sie sich heute zunehmend zur „individuellen Aufgabe“ (Jürgens 2012: 277f.). Die Reorganisation der Arbeitsverhältnisse, die Privatisierung und der Abbau des Sozialstaates und die Auflösung traditioneller Familienstrukturen verlangen individuelle Prävention und Selbstsorge (Jürgens Dezember 2010: 577). Ein permanenter Leistungsdruck entsteht, der mit einer Übertragung von Verantwortung an den Einzelnen einhergeht. Diese zunehmende Belastung bringen diverse AutorInnen in Verbindung mit dem Anstieg von psychosozialen Krankheiten wie Depressionen und Burnout (Ehrenberg 2008; Fisher 2015; Rosa 2011).

Gesamtgesellschaftlich betrachtet geht mit der makroökonomischen Entwicklung, also dem niedrigen Wirtschaftswachstum in den frühindustrialisierten Ländern und der Kapitalverschiebung von der Produktions- in die Zirkulationssphäre, die Zunahme der

10 Laut Statistischem Bundesamt ist der Anteil der Normalarbeitsverhältnisse von 77,7 Prozent im Jahr 1991 auf 66,8 Prozent im Jahr 2012 gefallen (BpB 31. Januar 2014).

11 So waren 1970 87,7 Prozent aller Männer erwerbstätig, während nur 45,9 Prozent der Frauen einem Beruf nachgingen. Im Jahr 2009 waren nur noch 75,3 Prozent der Männer, hingegen 65,1 Prozent der Frauen erwerbstätig (BpB 2010). 
sozialen Ungleichheit einher. ${ }^{12}$ Wie Thomas Piketty, der die Einkommens- und Vermögensverteilung anhand zahlreicher Daten seit dem 18. Jahrhundert untersucht hat, aufzeigt, steigt das Privatvermögen seit den 1970erJahren in allen Ländern Westeuropas und Nordamerikas an (Piketty 2016: 187-205). Indem die Kapitalrendite höher ist als der durchschnittliche Anstieg an Einkommen, werden KapitalbesitzerInnen tendenziell reicher und das Vermögen konzentrierter. In den Ländern des Globalen Nordens schrumpft durch die Verschiebung des Einkommen-Kapital-Verhältnisses die Mittelschicht (Nachtwey 2016: 151) und die Kluft zwischen Arm und Reich wird größer (Piketty 2016: 31). Da damit das in der Vergangenheit akkumulierte Vermögen, sprich das Erbe, wieder an Bedeutung gewinnt, verstetigt sich zugleich die Polarisierung der Sozialstruktur.

Eine ausgeprägte soziale Ungleichheit lässt sich auch für Hamburg, eine der reichsten Städte Europas, aufzeigen. Laut Ulrich Schneider vom Paritätischen Wohlfahrtsverband gibt es in Deutschland „keine Stadt, in der die soziale Spaltung schamloser stattfindet als in Hamburg" (zit. nach Carini 6. Juni 2012). Während Hamburg den höchsten Anteil einkommensreicher BürgerInnen aller Bundesländer hat (Pohl/Wicher 2013: 7) und die Anzahl an MillionärInnen in der Hansestadt stetig wächst - mittlerweile sollen es laut Zeitungsberichten über 40.000 sein (Mester 24. September 2014) -, nimmt zugleich trotz positiver wirtschaftlicher Gesamtsituation und leicht sinkender Arbeitslosenrate die Armut zu: Nachdem die Armutsquote 2008 auf 13,1 Prozent gesunken war, steigt sie nun wieder an. So haben 2015 15,7 Prozent der HamburgerInnen weniger als 60 Prozent des mittleren Einkommens aller Haushalte zur Verfügung (Der Paritätische Gesamtverband 2017: 5, 110). Auch stieg die Armutsgefährdungsquote ${ }^{13}$

12 Die Produktions- als auch die Zirkulationssphäre können im Kapitalismus nicht als unabhängig voneinander gedacht werden, sondern bilden eine Einheit: die des Produktionsprozesses des Kapitals. Die derzeitige Konfiguration des Kapitalismus verleitet jedoch gewisse politische Kräfte dazu, ihre Kritik allein auf die Zirkulations- und abstrakte Sphäre des Kapitalismus zu richten und damit einer personalisierten und potenziell antisemitischen Kapitalismuskritik Vorschub zu leisten. Wie Oliver Nachtwey zu Recht feststellt, läuft eine Kritik an der Verselbstständigung der Finanzmärkte fehl, verkennt sie doch, dass diese nur anwuchsen, weil keine ausreichenden Profite mehr in der Realwirtschaft erzielt werden konnten (Nachtwey 2016: 6of.).

13 Dieser Indikator gibt den Anteil der Bevölkerung an, der weniger als 6o Prozent des mittleren Einkommens verdient. im Zeitraum von 2005 bis 2016 für Haushalte, in denen die Person mit dem höchsten Einkommen maximal einen Realschulabschluss besitzt, von 36 Prozent auf 50 Prozent an (Statistische Ämter des Bundes und der Länder 2017). Besonders hart trifft die soziale Ungleichheit Menschen mit Migrationshintergrund: Nicht nur beträgt das Lohnniveau bei ihnen im Durchschnitt monatlich 1000 Euro weniger, auch lag 2016 die Arbeitslosigkeit bei HamburgerInnen mit Migrationshintergrund bei 16,1 Prozent und ohne Migrationshintergrund bei 5,7 Prozent (Meister/Niebuhr/Stöckmann 2017). Die guten Arbeitsmarktdaten und das Wirtschaftswachstum täuschen darüber hinweg, dass zunehmend mehr Menschen trotz Arbeit Probleme haben, ihre Lebenshaltungskosten, wie zum Beispiel die rasant steigenden Mieten, zu decken. Die soziale Polarisierung spiegelt sich auch in der Lohnquote Hamburgs, also dem Anteil der Arbeitseinkommen am Sozialprodukt. Diese liegt statt wie im bundesdeutschen Durchschnitt bei 63 Prozent in der Hansestadt nur bei 50 Prozent (Rose/Klages 2011: 17). „Wir müssen also feststellen, dass von Hamburgs hoher Arbeitsproduktivität und Wirtschaftskraft in ganz überwiegendem Maße die Unternehmer und Vermögenden profitieren, nicht jedoch die Arbeitnehmerinnen und Arbeitnehmer" (Rose/Klages 2011: 17).

Der Frust über schlechte Aushilfsjobs, niedrige Löhne und hohe Mieten kann sich jedoch politisch nur schwer artikulieren, da - wie seit längerem bekannt traditionelle Interessenvertretungen, insbesondere für Marginalisierte, an Bedeutung verloren haben (Merkel/ Petring 2011: 21). Gewerkschaftliche Vertretungen sind im Vergleich zum öffentlichen und industriellen Sektor - insbesondere in ungelernten Berufszweigen und den neuen Dienstleistungsjobs der Privatwirtschaft selten, die steigende Selbstständigkeitsquote negiert Interessensvertretungen und so sinkt der gewerkschaftliche Organisierungsgrad stetig. Auch den Parteien gelingt es immer weniger, Mitglieder aller Gesellschaftsschichten an sich zu binden. Die oft diagnostizierte Repräsentationskrise zeigt sich allerorts. Auch die Wahlbeteiligung ist davon betroffen. Diese nahm in Deutschland seit Jahren ab und stieg erstmals 2017 wieder an. ${ }^{14}$ Wie Wolfgang Merkel und Alexander Petring nachgewiesen haben, zeichnet sie sich durch eine zunehmende soziale

14 Nachdem die Wahlbeteiligung seit 1998 sank, stieg sie zur Bundestagswahl 2017 wieder, auf 76,2 Prozent. Die höchste Beteiligung an einer Bundestagswahl in der Nachkriegszeit fand 1972 statt, als 91,1 Prozent der Wahlberechtigten wählen gingen. 
Selektivität aus, das heißt der Anteil an NichtwählerInnen unter den Geringverdienenden und Menschen mit keinem oder geringem Bildungsabschluss stieg in den vergangenen Jahren und ist überproportional hoch (Merkel/Petring 2011: 8-13). Auch auf Menschen mit Migrationshintergrund, von denen viele nicht wählen gehen dürfen, trifft die Diagnose der mangelnden politischen Repräsentation zu. In Billbrook, dem Stadtteil mit dem höchsten Anteil an Menschen mit Migrationshintergrund (84,9 Prozent), gingen bei der Bürgerschaftswahl in Hamburg 2015 nur 26,2 Prozent der Berechtigten wählen (Statistisches Amt für Hamburg und Schleswig-Holstein 15. Februar 2015). Zwar haben sich gesamtgesellschaftlich die individuellen Partizipationschancen „jenseits parlamentarische[r] Formen“ wie Bürgerinitiativen erhöht (Nachtwey 2016: 92), aber solche alternativen Beteiligungsformen zeichnen sich im Vergleich zu den Wahlen durch eine noch höhere soziale Selektivität aus (Merkel/Petring 2011: 21).

Doch trotz dieser Repräsentationskrise, die insbesondere auf marginalisierte Bevölkerungsteile zutrifft, gibt es in Deutschland keine Riots von rassifizierten Jugendlichen, wie wir sie von Frankreich, England und den USA kennen. Zwar sind auch für Hamburg Ausschreitungen von migrantischen Jugendlichen bekannt, die aufgrund von rassistisch wahrgenommenen Polizeikontrollen und dem „brutale[n]“ und „unverhältnismäßige[n] “ Vorgehen der Polizei (Mücke/ Rinn 2016: 115) im Jahr 2013 aufflammten. Warum das Ausmaß und die Intensität solcher Ausschreitungen vergleichsweise gering sind, kann hier nur spekulativ beantwortet werden. Nimmt man Clovers These vom Zusammenhang von Produktion, Zirkulation und Konfliktartikulation ernst, zeigt sich eine deutsche Besonderheit. Deutschlands Wirtschaft ist im Vergleich $\mathrm{zu}$ anderen frühindustrialisierten Ländern immer noch stark von der Produktion und mittelständischen Betrieben geprägt (Sassen 2015: 160). ${ }^{15}$ Diese Wirtschaftsweise, die mit einer geringeren sozialen Ungleichheit einhergeht und die zugleich von einem immer noch umfassenderen Wohlfahrtsstaat als etwa in den angloamerikanischen Ländern gestützt wird, führt womöglich zu einer weniger tiefgreifenden Marginalisierung von Teilen der Bevölkerung.

15 In Deutschland liegt der Anteil an Finanzvermögenswerten bei 175 Prozent des Bruttoinlandsprodukts, während er in den USA 450 Prozent und in Großbritannien 440 Prozent und in der EU durchschnittlich 356 Prozent beträgt (Sassen 2015: 160).
Makrostrukturell lässt sich eine Verschärfung der sozialen Ungleichheit für Deutschland und Hamburg feststellen, doch die Protestierenden gegen den G20-Gipfel gehören, wie Umfragen zeigen, weniger marginalisierten Teilen der Bevölkerung, sondern überwiegend der Mittelschicht an. ${ }^{16}$ Auch wenn Angehörige der Mittelschicht durch ihre akademischen Abschlüsse statistisch gesehen seltener arbeitslos werden, sind sie nicht mehr vor Prekarisierung und unsicheren Zukunftserwartungen gefeit. Insbesondere in Hamburg tritt seit Jahren eine urbane soziale Bewegung auf, die soziale Gerechtigkeit und Mitbestimmung nicht nur abstrakt thematisiert, sondern ganz konkret in Bezug auf die eigene Lebenswirklichkeit einfordert. Das 2009 entstandene Recht auf Stadt-Netzwerk ist ein deutlicher Ausdruck dieses sich artikulierenden sozialen Konfliktes. Die beteiligten Gruppen skandalisieren nicht nur die auch für Angehörige der Mittelschicht extrem hohen Mieten, sondern sie kritisieren zudem eine Stadtentwicklung, die sich aus der Perspektive der stadtpolitischen AktivistInnen mehr nach Interessen der InvestorInnen als der Hamburger BürgerInnen richtet. In den Auseinandersetzungen der vergangenen Jahre wurde zugleich deutlich, dass zwar städtischer Protest laut organisiert werden kann, aber die realen Einflussmöglichkeiten auf die Stadtentwicklung mehr als beschränkt sind. Neben dem Recht auf Stadt-Netzwerk ist es auch der Rote Aufbau Hamburg, der für eine Verschiebung im Spektrum der politischen Mobilisierung steht. Diese aus einer Spaltung 2015 hervorgegangene Gruppe ist klar dem antiimperialistischen Spektrum zuzuordnen und hat laut Verfassungsschutzbericht steigende Mitgliederzahlen zu verzeichnen (Landesamt für Verfassungsschutz 2017: 103f.). Auffällig an dieser Gruppe ist eine Rhetorik, in der man sich klar von einer Linken abgrenzen möchte, die aus „Wohlstandslinken“ und AkademikerInnen bestehe und die nur Theoriearbeit leiste (Roter Aufbau Hamburg 2015). Sie wollen keine StudentInnengruppe sein, sondern sehen sich im „Klassenkampf“ und in der „revolutionären Praxis“ - so wird es im Selbstverständnis deutlich - vielmehr als Teil der Arbeiterklasse und der Unterschicht (Roter Aufbau Hamburg 2015). Dass eine aktionistisch auftretende

16 Laut der Befragung durch das Institut für Protestund Bewegungsforschung verfügten bei den Demonstrationen gegen den G2O-Gipfel in Hamburg mehr als 6o Prozent der Teilnehmenden über einen Hochschulabschluss - im Vergleich zu 15 Prozent in der Gesamtgesellschaft (Institut für Protest- und Bewegungsforschung November 2017). 
Gruppe, deren Sprecher einen Migrationshintergrund aufweist, einerseits sich nicht vorrangig aus bürgerlichen Kreisen rekrutiert und andererseits explizit die soziale Frage stellen will, kann nur als Indikator eines zunehmenden sozialen Konflikts gedeutet werden. Sowohl von diesem Protestmilieu als auch von den Recht auf Stadt-Gruppierungen, deren AktivistInnen überwiegend aus der Mittelschicht stammen, wird dabei der Kapitalismus als Ursache der gesellschaftlichen Krise benannt. Diese Problemdiagnose teilen nicht nur die benannten Hamburger politischen Zusammenhänge, sondern auch der Großteil der Protestierenden gegen den G20-Gipfel.

\section{Ideelle (Hinter-)Gründe für die Riots}

Obwohl die Großdemonstration am Samstag unter dem Motto „Für eine grenzenlose Solidarität“ sehr heterogen war, da unterschiedliche politische Spektren mobilisiert hatten, kamen die 70.00o Menschen nicht allein aufgrund ihrer Gegnerschaft zu Trump oder Putin zusammen. Die Sorge um den Klimawandel und die Ablehnung der herrschenden Profitlogik und Wirtschaftsordnung können, wie zahlreiche Transparente, Sprechchöre und mittlerweile eine Erhebung belegen, als zentrale Beweggründe der Protestierenden gelten. Gemäß der Studie des Instituts für Protest- und Bewegungsforschung stimmten mehr als drei Viertel der befragten DemonstrantInnen „überwiegend“ oder „voll und ganz“ der Aussage zu, dass der Kapitalismus überwunden werden müsse (Institut für Protest- und Bewegungsforschung, November 2017). Wie umfassend die Kritik ist, zeigt ein internationaler Aufruf zu den G20-Protesten, der unter anderem von deutschen, spanischen, griechischen, italienischen und argentinischen Gruppen unterzeichnet wurde:

"[W]e will be loud against the exploitation of labour and the destruction of nature in the name of profit, and loud for equal rights and gender equality. Let them talk about their "migration crisis"; we will speak up for open borders and against racism; we will address the systematic production of poverty and war that forces millions of people to move" (NoG2o International 2017).

In der Kritik der Protestierenden zeigt sich darüber hinaus, dass die Kapitalismuskritik grundlegender geworden ist. Die zahlreichen Krisenherde und Naturkatastrophen nähren die Angst vor einem bevorstehenden Untergang der Welt.

„Diese G2o repräsentieren uns nicht! Sie haben keine Lösungen für die realen Probleme unserer Welt. Ihr Wirt- schaftssystem ist ein einziger Wahnsinn: ohne Respekt vor der Zukunft, der Natur und dem Recht aller Menschen auf ein gutes Leben in Würde und Freiheit. Sollen wir zuschauen, wie sie unsere Welt zugrunde richten?" (BlockG2o 2. April 2017)

Diese sich hier andeutende apokalyptische Grundstimmung spitzt sich im Insurrektionalismus militant $\mathrm{zu}$ und sollte näher betrachtet werden, wenn man die ideellen Beweggründe für die Ausschreitungen nachvollziehen möchte. Diese anarchistische Strömung mit deutlich nihilistischen Tendenzen, die in der französischen, italienischen und griechischen Linken relativ stark ist und die auch unter deutschen AnarchistInnen an Einfluss gewinnt, sieht sich, wie es in „Der kommende Aufstand“ (Comité Invisible 2010) heißt, mit einer drohenden „Auslöschung der Zivilisation“ konfrontiert (ebd.: 61). Im Klimawandel, in Bürgerkriegen und der zunehmenden Inwertsetzung des eigenen Selbst und gesellschaftlicher Teilbereiche antizipieren sie die bevorstehende Selbstzerstörung und die totale Katastrophe. Der fehlende Zukunftsoptimismus führt dazu, dass jegliche Hoffnung auf eine Besserung gesellschaftlicher Verhältnisse, eine auf Reformation zielende Politik und somit auch das Formulieren von Forderungen aufgegeben wurden. Infolgedessen stellen sie nicht nur die repräsentative Demokratie infrage und lehnen eine Kooperation mit institutionalisierten Interessensvertretungen wie Gewerkschaften ab, sondern sie vertrauen mitunter auch nicht auf soziale Bewegungen als soziale Kraft (Gelderloos 2013: 12f. Comité Invisible 2010: 82; translationcollective 8. März 2010). Als Beweis für deren Versagen gilt ihnen eine Identitätspolitik und die Kommerzialisierung von feministischen und ökologischen Positionen oder von subkulturellen Milieus (CrimethInc. 2010; Dragonowl 2015). ${ }^{17}$ Somit wenden sich die InsurrektionalistInnen häufig dem militanten Aktionismus zu, um der gefühlten Ohnmacht und Apathie eine unmittelbare emotionale Resonanz entgegenzusetzen. Durch eine direkte Konfrontation soll die Meuterei und die Subversion der „Ausgebeuteten und Ausgeschlossenen“ verbreitet werden, wobei die gewaltvollen Auseinandersetzungen weniger einen militärischen Angriff bedeuten, sondern die gesellschaftliche Normalität, etwa in Form von Sabotageakten, durchbrechen und die Wirtschaft lähmen sollen

17 Somit stellt sich ebenfalls die Frage, inwiefern der Insurrektionalismus auch als eine Reaktion auf das Ausbleiben kollektiver sozialer Kräfte und dem Mangel an utopischen Ideen interpretiert werden kann. 
(translationcollective 8. März 2010). Ziel ist es, Bilder der Zerstörung zu produzieren, um so die Widersprüche und Krisenmomente der Welt sichtbar werden $\mathrm{zu}$ lassen.

Auch wenn die soziale Ungleichheit vergleichsweise gering ist und die sozialen Konflikte in Deutschland weniger drastisch als anderswo ausfallen, ist die Teilhabe am Wirtschaftswachstum und an politischen Interessensvertretungen in den vergangenen Jahren zunehmend schwieriger geworden, wobei die beschriebene Repräsentationskrise nicht allein die marginalisierten und/oder rassifizierten Bevölkerungsgruppen betrifft. ${ }^{18}$ Auch die Diskurse und Praktiken eines Teils der radikalen Linken und speziell die zunehmende Bedeutung des Insurrektionalismus sind Ausdruck dieser Krise. Einen expliziten Zusammenhang zwischen der derzeitigen Krise politischer Repräsentation und den Riots stellt $\mathrm{AK}$ Thompson her, indem er ausgehend von den Ausschreitungen der Antiglobalisierungsbewegung 1999 in Seattle die Attraktion der Riots für weiße Mittelschichtsjugendliche erklärt (Thompson 2010). Riots böten eine Rückkehr zum Politischen an, indem sie die Möglichkeit schaffen, die unmittelbare Realität $\mathrm{zu}$ produzieren (Jasper/Thompson 2016: 225): „At their best, riots remind us that the basic unit of politics is not representation" (ebd.: 222).

Jugendliche sowohl aus marginalisierten Bevölkerungsteilen als auch aus der Mittelschicht teilen Gefühle der Ohnmacht, des Pessimismus und das vage Gefühl, dass es ihnen später, wenn sie erwachsen und berufstätig sind, nicht besser gehen wird als ihren Eltern. ${ }^{19}$ Sie sehen sich in ihrem Leben, wenn auch in deutlich unterschiedlicher Intensität, mit einer mangelhaften sozialen Absicherung und eingeschränkten politischen Einflussmöglichkeiten konfrontiert.

18 Bezeichnenderweise mobilisierte das kommunistische UmGanze-Bündnis während des G2o-Gipfels am Freitagmorgen zu einer Blockade des Hafens, da man sich erhoffte, „trotz unserer gesellschaftlichen Bedeutungslosigkeit, das Kapital da zu treffen, wo es ihm auch weh tut" (UmsGanze 2017). Die hier auftretenden ProtestakteurInnen wissen um das Problem, keinen Streik realisieren zu können, da sie nicht in Betrieben, also in der Produktion, tätig sind.

19 Die Trennung in marginalisierte (und häufig rassifizierte) Menschen auf der einen und Linken aus der Mittelschicht auf der anderen Seite ist natürlich eine schematische (und auch wenig wünschenswerte) Darstellung. Aber da sich viele linke Zusammenhänge durch eine gewisse, von einer weißen Mittelschicht geprägten, Homogenität auszeichnen, sei diese Zuspitzung erlaubt.
Doch weder die ideellen oder emotionalen Beweggründe noch die soziostrukturellen Ursachen liefern eine vollständige Erklärung für den Ausbruch von Riots. Vielmehr gibt Javier Auyero zu bedenken, dass "the identification of larger factors related to the occurrence of riots is different from the examination of the chain of causalities that produce them" (Auyero 2001: 35). Aus einer relationalen Perspektive heraus soll daher zuletzt die konkrete Konstellation in Hamburg in den Blick genommen werden, in der die ProtestakteurInnen zu den Ordnungskräften standen. Insbesondere die Dynamik und die damit einhergehenden Affekte, die zur Zuspitzung der Konfliktsituation beigetragen haben, bedürfen einer näheren Betrachtung.

\section{Der Ausnahmezustand als Schmelztiegel}

Obwohl die Polizei mit über 30.000 Sicherheitskräften (dpa 7. September 2017) - und damit mit allen zur Verfügung stehenden PolizistInnen Deutschlands (ZDF 9. Juli 2017) $)^{20}$ - vor Ort war, konnte sie zwar die sichere Durchführung des Gipfels gewährleisten, jedoch die immensen Ausschreitungen nicht verhindern. Das lag weder an der übergroßen Zahl von Autonomen noch an einem Mangel an polizeilicher Kompetenz, Personal oder Technik. Die Polizei hatte vielmehr Probleme, durchgängig die Kontrolle über die Stadt zu behalten, da sie auf eine Vielzahl von ProtestakteurInnen traf, die aus radikalen Militanten aus Deutschland und dem Ausland, ${ }^{21}$ aus organisierten Linken unterschiedlicher politischer Spektren, aus linksbewegten BürgerInnen und von dem Polizeieinsatz genervten AnwohnerInnen sowie eher szenefremden Jugendlichen bestanden und die zeitweise zu einer Protestmasse verschmolzen.

20 Zumindest ohne in anderen Teilen von Deutschland einen polizeilichen Notstand auszulösen.

21 Dass manche der ausländischen DemonstrantInnen den deutschen Autonomen in Sachen Militanz den Rang ablaufen, sollte nicht überraschen. In vielen europäischen Ländern existiert ein höheres Eskalationsniveau, das auch mit den angereisten Militanten nach Hamburg kam. Beispielsweise wurden in Frankreich eine Woche nach dem G20-Gipfel bei Ausschreitungen an einem Wochenende knapp 900 Autos angezündet (afp 15. Juli 2017). Zudem herrschen in vielen südeuropäischen Ländern prekäre Lebensund Arbeitsbedingungen. So besteht unter Jugendlichen eine immens hohe Arbeitslosigkeit und die staatlichen sozialen Absicherungssysteme funktionieren nur eingeschränkt. Insofern trugen die ausländischen AktivistInnen förmlich die wirtschaftliche und politische Misere, die viele Länder Europas erreicht hat und von der Deutschland weitgehend verschont blieb, nach Hamburg. 
Die immense Eskalation am Freitagabend im Schanzenviertel, bei der auch Plünderungen stattgefunden haben und die durch den Einsatz eines schwerbewaffneten Sondereinsatzkommandos ihr Ende fand, wurde von einer sozial heterogenen Gruppe von Menschen bestimmt, die weit über das klassisch linke, häufig mittelschichtsgeprägte Spektrum hinausreichte. HamburgerInnen kennen diese ritualisierten Entladungen nach Schanzenfesten oder nach dem 1. Mai nur zu gut. Mit immer späterer Stunde und steigendem Alkoholpegel wird das Geschehen regelmäßig von jungen Männergruppen bestimmt. Auch am Freitagabend ereignete sich eine ähnliche Situation. Zuerst wurden viele ProtestakteurInnen durch den Einsatz von Wasserwerfern und polizeilichen Maßnahmen in das Schanzenviertel getrieben. Anschließend fingen 200 bis 300 Autonome an, Barrikaden zu bauen und Schaufester einzuwerfen (Lotzer 2018b: 132). Für die meisten der InitiatorInnen waren die Aktionen vermutlich ein symbolischer Angriff auf das herrschende System, indem Privateigentum und die öffentliche Ordnung angegriffen wurde. Doch innerhalb kürzester Zeit gesellten sich zu den militanten Linken zahlreiche, zum Teil auch migrantische Jugendliche, die kaum einen oder keinen Bezug zur linken Szene haben. Das erklärt auch, warum die einen die geplünderten Flachbildmonitore nach Hause trugen, während die anderen die teuren Apple-Produkte ins Feuer schmissen. Wie die „Panorama“-Sendung auf ARD zu berichten weiß, trafen an diesem Freitagabend „Autonome, Junggesellenabschiede, G20-Gegner, Anwohner, VorstadtJugendliche, Krawalltouristen und Partygänger [...] aufeinander - und auf die Polizei“ (Reisin 20. Juli 2017). Dass sich die „Schlacht auf der Schanze“ nicht auf die Handlung einiger Autonomer reduzieren lässt, bestätigt selbst die Polizei, als sie bekannt gab, dass die Situation am Freitagabend auch aufgrund der Teilnahme zahlreicher Trittbrettfahrer nicht mehr kontrollierbar gewesen sei (Hamburger Abendblatt 19. Juli 2017). Auch in bewegungsnahen Publikationen kann man lesen, dass die Ausschreitungen am Freitagabend besonders waren und zwar nicht, weil einige InsurrektionalistInnen die Stadt unsicher gemacht haben (Doell 2018: 80), sondern weil der „allergrößte Teil der Teilnehmenden [...] weder Anarchist*innen noch fremde aus Südeuropa, sondern ganz normale Leute aus Hamburg“ waren (CrimethInc. Ex-Workers'Collective 2018: 50). Doch wie kam es, dass, wie es in der vom Institut für Protest- und Bewegungsforschung herausgegebenen Protestbeobachtung heißt, „Männer unterschiedlicher
Milieus und Schichten brennende Barrikaden bauten und die Polizei durch Flaschen- und Steinwürfe auf Abstand hielten"? Wie kam diese Phalanx zustande? Die Ausschreitungen am Freitagabend können ohne sozioökonomische und ideelle Gründe nicht erklärt werden. Doch auch wenn diese Ursachen notwendige Bedingungen darstellen, lassen sie sich nicht darauf reduzieren. Die Eskalation erklärt sich vor allem aus der Situation heraus, für die die Dynamik während der Gipfeltage prägend war und die es genauer zu untersuchen gilt.

Schon im Vorfeld missfiel es Teilen der Hamburger Bevölkerung, dass ihnen keinerlei Mitbestimmungsmöglichkeit über die Entscheidung, den Gipfel in Hamburg auszurichten, eingeräumt wurde. Auch die 40 Quadratkilometer große Demonstrationsverbotszone und der drohende polizeiliche Ausnahmezustand verstimmten viele, insbesondere die linksbewegten HamburgerInnen. In Erwartung auf großräumige Absperrungen, polizeiliche Kontrollen und permanente Hubschrauberpräsenz fragten stadtpolitische Initiativen im Vorfeld: „Was machen die aus unserer Stadt?“ (St. Pauli Selber Machen 29. Mai 2017). Die Messehallen, in denen das Treffen der Staatschefs abgehalten wurde, liegen unmittelbar in der Nähe zu den Stadtvierteln Sternschanze und St. Pauli. Vermutlich gibt es in ganz Deutschland kein städtisches Gebiet, in dem in einer so hohen Konzentration linksbewegte und linksradikale BürgerInnen wohnen. ${ }^{22}$ Die Austragung des G2O-Gipfels in unmittelbarer Nähe zu symbolisch als „linke Kieze“ aufgeladenen Stadtteilen, wurde von vielen AnwohnerInnen als arrogante Machtdemonstration, von einigen Linksradikalen sogar als gezielte Provokation wahrgenommen.

Durch die Ausrichtung des Gipfels in einer Metropole und vor dem Hintergrund der permanenten Terrorismusgefahr sahen sich die politischen Verantwortlichen genötigt, die Sicherheit der mächtigsten Regierungschefs mittels massiver Polizeipräsenz und der partiellen Einschränkung von Grundrechten zu gewährleisten. Viele AnwohnerInnen, die niemals aus Protest zu einem Gipfel fahren würden, störten sich aber sukzessive an der polizeilichen Belagerung und

22 Die Ergebnisse der letzten Bürgerschaftswahl verdeutlichen diese These: Während in St. Pauli 4,1 Prozent und in der Sternschanze gerade mal 2,9 Prozent für die CDU stimmten, war die stärkste Partei in beiden Stadtteilen mit fast 30 Prozent die Linkspartei, dicht gefolgt von den Grünen (Statistisches Amt für Hamburg und Schleswig-Holstein 15. Februar 2015). 
den immensen Kosten, die für die Ausrichtung des Treffens veranschlagt wurden. Wenn man sich etwa den Dokumentarfilm „Vor dem Knall“ anschaut, wird deutlich, inwiefern der polizeiliche Ausnahmezustand zur Politisierung von Teilen der Bevölkerung geführt hat und weshalb sie zu GipfelgegnerInnen wurden (Granvista Media 2018). Diese lokale Konstellation erklärt die große Unterstützung der ansässigen Bevölkerung für die Gipfelproteste. Darüber hinaus waren die Aktionen gegen den G20-Gipfel logistisch sowie infrastrukturell bestens aufgestellt und konnten meist eine lokale Verankerung aufweisen. Hamburg hat eine lange Geschichte an linker Protestkultur und linken Strukturen vorzuweisen, und so öffneten während der Gipfeltage über zwanzig Orte ihre Türen, um den AktivistInnen Erholung, Rückzug und Verpflegung anzubieten (Mullis 20. Mai 2018: 35). Das Ausmaß der Proteste lässt sich ohne diese linke Infrastruktur nicht verstehen. ${ }^{23}$ In Anbetracht dieser speziellen Ausgangslage erklärt sich auch, warum sich die Gipfelproteste in Hamburg nicht einfach auf eine symbolische Kritik an der kapitalistischen Weltordnung und ihren RepräsentantInnen beschränkte. Der Konflikt zwischen Protestierenden und Herrschenden mündete gleichsam in eine konkrete Auseinandersetzung um eine territoriale Kontrolle, bei der um die Macht über die Straßen und Viertel gerungen wurde. Die während des Gipfels stattgefundenen Demonstrationen, das Cornern, die Platzbesetzungen, Techno-Raves und Riots waren Aneignungen des urbanen Raums, die der Selbstermächtigung dienten.

Die schon im Vorfeld durch die beschriebene lokale Konstellation angeheizte Dynamik zwischen AnwohnerInnen und Protestierenden einerseits und der Polizei andererseits spitzte sich während der Gipfeltage stetig zu. Den Vorhaben der AktivistInnen, in Camps zu übernachten oder auf der Straße zusammenzukommen, wurde mit einer Machtdemonstration in Form eines massiven Polizeiaufgebots begegnet. Dass auch die PolizistInnen durch Überarbeitung an das Ende ihrer Kräfte kamen und dies eine besonnene Handhabung von konfliktiven Situationen kaum beförderte, ist selbstredend. Einige AutorInnen diagnostizieren jedoch nicht nur situative Grenzüberschreitungen,

23 Diese lokale Konstellation - sprich die Unterstützung der Protest durch Teile der lokalen Bevölkerung und die linke Infrastruktur - erklärt auch den Unterschied zu Protesten inklusive der Ausschreitungen während der Gipfelproteste in Heiligendamm 2007. sondern sprechen aufgrund diverser Verstöße gegen die Versammlungs- und Pressefreiheit und polizeilicher Übergriffe von einer Suspendierung der Grundrechte, die den Rechtsstaat infrage stelle (Mullis 2o. Mai 2018; Loick 28. Juli 2017). ${ }^{24}$ Viele der Protestierenden sind es gewohnt, dass ihre Blockadeversuche aufgrund effektiver Polizeiarbeit symbolisch bleiben. Jedoch trübten die wahrgenommenen Rechtsbrüche und willkürlichen Gewaltanwendungen für abertausende Protestierende den Glauben an Recht und Gesetz. So führten die zahlreichen Erfahrungen mit einer repressiven Polizei dazu, dass ein Verständnis für eine direkte Konfrontation mit der Polizei über die Tage anwuchs. Immer wieder scheiterte die Polizei daher mit ihrer Aufforderung an die DemonstrantInnen, sie sollen sich von den wenigen Menschen räumlich distanzieren, die mit Flaschen und Steinen auf die Polizei losgingen. Eine klare Trennung zwischen friedlichen DemonstrantInnen und gewalttätigen RandaliererInnen hat es in Hamburg nicht gegeben, vielmehr war der Übergang zwischen den Gruppen fließend. Wie auch Fox Piven betont, ist Gewalt in der Verweigerung der institutionellen Regeln zentral (Fox Piven 2012: 25), weshalb sie $\mathrm{zu}$ dem Schluss kommt "that violence and the threat of violence are complexly intertwined with the efforts of social movements to exercise power" (Fox Piven 2012: 27). Damit wird deutlich, dass die Trennung zwischen RandaliererInnen und sozialen Bewegungen zuvorderst eine imaginäre ist.

Die Dynamik der Proteste spitzte sich über die Tage sukzessive zu. Laut Daniel Mullis lässt sich die Situation in Hamburg als Ausnahmezustand beschreiben. Demnach führten die polizeilichen Maßnahmen zu einem faktischen „Aussetzen von Recht“ und einer „Produktion von Unsicherheit und Angst", mit denen Protest unterbunden und delegitimiert werden sollte (Mullis 20. Mai 2018: 33). Während die Polizei den Ablauf des Gipfels und die Sicherheit der Regierungschefs mit

24 So hat auch das Komitee für Grundrechte und Demokratie auf der Grundlage von 43 DemonstrationsbeobachterInnen einen Bericht vorgelegt (Komitee für Grundrechte und Demokratie e.V. 2017), in dem Verstöße gegen die Versammlungs- und Pressefreiheit dokumentiert sind. Insbesondere der Umgang mit dem Camp in Entenwerder, die polizeiliche Auflösung der „Welcome to hell“-Demonstration, die Festsetzung zahlreicher DemonstrantInnen am Freitagmorgen am Rondenbarg und die wiederholte Vertreibung von Journalisten vom Demonstrationsgeschehen zogen starke Kritik auf sich, weshalb das Komitee einen Untersuchungsausschuss einforderte. 
einer physischen und technischen Übermacht, sprich mit einer robusten und zuweilen brachialen Ausführung des Gewaltmonopols, gewährleistete, stritten die GipfelgegnerInnen aus ihrer Sicht um grundsätzliche demokratische Fragen: „Wer darf wo schlafen, essen, trinken, protestieren, sich den öffentlichen Raum aneignen?“ Wie aus dem Dokumentarfilm „Festival der Demokratie“ (Rilrec 2018) hervorgeht, führten die tagelangen Auseinandersetzungen mit der Polizei im Laufe der Zeit jedoch dazu, dass die Protestierenden zunehmend ihre Angst vor den Ordnungskräften verloren.

Als am Freitagabend viele ProtestakteurInnen, die den ganzen Tag über erfolglos versucht hatten, den Gipfel zu beeinträchtigen, in das Schanzenviertel getrieben wurden und sich dann die Polizei zurückzog und den Protestierenden das Viertel überließ, entflammten stundenlange Riots. Seit Tagen und Wochen wurde der öffentliche Raum durch polizeiliche Maßnahmen dominiert, Proteste wurden eingeschränkt, Schlafcamps aufgelöst und der Angriff auf die Demonstration „Welcome to hell“ am Vorabend hatte bei allen Anwesenden einen großen Frust über die Polizei ausgelöst und nun bestand die Möglichkeit, „sich für Momente der aufgezwungenen Ohnmacht entledigen zu können“ (Lotzer 2018a: 95). Der Riot am Freitagabend, bei dem die Polizei temporär die Kontrolle über das Viertel verlor, kann somit als Umkehrung des Ausnahmezustands gelesen werden. Der Rausch entstand (und hierin liegt auch eine Analogie zu den Riots in den marginalisierten Vierteln) durch die Zurückweisung der Polizei und der Ausübung der sonst nicht verfügbaren Macht über ein Territorium. Diese Umkehrung des Ausnahmezustands, die zugleich in einen führt und der für verängstigte AnwohnerInnen und Außenstehende kaum zu begreifen ist, spiegelt sich in folgender Beschreibung der Situation hinter den Barrikaden:

„Die Atmosphäre war entspannt. Leute standen gemeinsam auf der Straße, bewunderten die Feuer, unterhielten sich, es gab Getränke und Essen. Außerhalb, dort wo die Polizei die Straßen kontrollierte, war die Hölle aus Gewalt, Chaos und Angst. Hier, wo sie die Kontrolle verloren hatte, erfuhren wir die ersten friedlichen Momente der letzten Tage" (CrimethInc. ExWorkers'Collective 2018: 49).

Während die Riots dazu führten, dass die Proteste gegen den G2O-Gipfel in den Medien und in der politischen Öffentlichkeit zwar wahrgenommen, diese aber zugleich in ein schlechtes Licht gerückt wurden, scheinen die positiven Effekte - ohne die ein wiederholtes
Zustandekommen dieser Praktiken nicht erklärbar wäre - somit bei den Randalierenden zu liegen. Wie Armin Nassehi in der Zeit treffend beschreibt, bestehe die Attraktion körperlicher Gewalt darin, „dass sie die Zeit verkürzt, dass sie unmittelbare Wirkung zeitigt“. Der Riot bietet „für einen Moment [...] totale Macht, Durchsetzungsfähigkeit und Autonomie“ (Nassehi 13. Juli 2017). Ist die politische Wirkmacht schon beschränkt, und das lässt sich in Bezug auf die Gipfelproteste durchaus konstatieren, lässt sich durch das Erfahren spektakulärer Situationen und intensiver Gefühle offenbar eine persönliche Befriedigung erreichen.

Neben den ideellen Beweggründen sind es somit vor allem die gemeinsam erlebten Erfahrungen und Emotionen, die ein kurzlebiges Kollektiv herzustellen vermögen. ${ }^{25}$ Wie ein solches in Hamburg funktioniert hat, konnte man in der Neuen Zürcher Zeitung lesen: „Es gibt, wie im Hooligan-Milieu, erfahrene Leute, die den Ton angeben und die Gruppe koordinieren. Die einen brechen Steine aus dem Pflaster und zerbrechen diese in wurffähige Brocken, die anderen fahren die Geschosse in Einkaufswagen oder Mülltonnen dorthin, wo sie gebraucht werden. Und eine dritte Gruppe errichtet im Hintergrund Barrikaden, die dann in Brand gesetzt werden“ (Serrao 11. Juli 2017). Für die Koordinierung des Riots, also der Realisierung einer kollektiven Handlung, bedarf es eines, wenn auch kurzweiligen Gemeinschaftsgefühls und einer geteilten Solidarität, wie Reicher und Stott gezeigt haben (Reicher/ Stott 2011: 41, 43). Liest man die Einschätzungen von Menschen, die am Freitagabend im Schanzenviertel zugegen waren, wird deutlich, dass die Teilnehmenden vor allem einen affektiven Gewinn aus den Vorkomm-

25 Emotionen spielen aber nicht nur bezüglich der Riots, sondern auch allgemein für soziale Bewegungen eine wichtige Rolle (siehe Jasper 2011). Wenn sich in Hamburg Zehntausende Protestierende treffen, um ihren Unmut über den polizeilichen Ausnahmezustand und die Politik der G2o zum Ausdruck zu bringen, dann kommen sie nicht nur zusammen, um diesen nach außen zu tragen. Der Konvent der G20-KritikerInnen hat darüber hinaus einen wichtigen, nach innen gerichteten Effekt: Menschen aus unterschiedlichen Orten und Zusammenhängen lernen sich kennen und erleben eine gemeinsame Zeit. Sie kochen, diskutieren und demonstrieren zusammen; sie organisieren sich. Der Gipfel stärkt den Zusammenhalt und den Austausch innerhalb der Bewegung. Das Gefühl, Teil einer kritischen Masse zu sein, führt zu einer Bestätigung der eigenen linken Identität und zu einer Selbstermächtigung, die der oft im Alltag erlebten Ohnmacht entgegensteht. 
nissen zogen. ${ }^{26}$ Alle seien „sehr nett zueinander“ und „hilfsbereit“ (Melchers 20. Juli 2017), es sei ein großes „Fest“ mit „euphorischer Stimmung“ (Rauschenbach 24. August 2017) gewesen. Insbesondere die Verteilung der im Supermarkt geplünderten Waren, allen voran Tabak, Alkohol und Süßigkeiten, habe zur guten Stimmung beigetragen. Der daraus „resultierenden Magie des Augenblicks“, der großen „KomplizInnenschaft“ hätte „sich kaum jemand entziehen können“. ${ }^{27}$ Aber nicht nur die „helle Geschwisterlichkeit“ wird von einigen der Anwesenden geschätzt, sondern die emotionale Energie der „Unmittelbarkeit der Präsenz, sich selbst und den Anderen gegenüber" (Imaginäre Partei 2018: 119). So heißt es in einer die Riots glorifizierenden linken Publikation: „Diese Freude, diese Angst und die Intensität hat uns soviel lebendiger gemacht, als der reguläre Gang der Dinge im kapitalistischen Alltag“ (Imaginäre Partei 2018: 118f.). Der Riot ist somit weniger ein Akt, mit dem politisch etwas erreicht werden soll, sondern stellt vielmehr ein soziales Ereignis dar.

Die anwesenden Protestierenden teilten zwar eine gewisse Unzufriedenheit und das Gefühl der sozialen Unsicherheit, aber um ein kollektives linkes Subjekt handelte es sich nicht. Neben politisch überzeugten Linksradikalen, die häufig der Mittelschicht entstammen, standen von dem Polizeieinsatz genervte linksliberale AnwohnerInnen und migrantische als auch kaum politisierte Jugendliche. Die heterogene Mischung aus sozial und politisch Unzufriedenen wurde erst durch den Konflikt mit der Polizei zusammengebracht, wodurch vorübergehend eine Koalition entstand, die politische Spektren und soziale Schichten übergreifend war. Nicht zufällig war der wohl meistgerufene Sprechchor über die Tage hinweg, also der kleinste gemeinsame Nenner, der alle vereint: „Ganz Hamburg hasst die Polizei“. Für Clover lässt sich ein Riot nicht ohne Polizei denken, sie ist nicht nur das vereinigende Gegenüber, sondern integraler Bestandteil eines Riots.

26 Vollständigkeitshalber soll angefügt werden, auch wenn das nicht Gegenstand des Artikels ist, dass viele Anwesende und AktivistInnen die Ausschreitungen im Allgemeinen oder einzelne für Menschen gefährliche Aktionen höchst problematisch fanden und es schon vor Ort als auch im Nachgang diverse Auseinandersetzungen über die Gewaltfrage gab.

27 Diese Einschätzung kann man einer anonymen Broschüre entnehmen, die in Szenekreisen erschienen ist und die die Ausschreitungen wohlwollend reflektiert (Anonym 2017: 14ff.). Teile der Aufzählung stammen von crimethInc (CrimethInc. Ex-Workers'Collective 2018: 50).
Er spricht von einer Riot-Polizei-Dialektik und bemüht dabei folgendes Statement aus einer linken Zeitschrift, das in Bezug auf die Riots in England entstand, das aber ebenso für die Situation in Hamburg gelten kann:

„It is a perversity of a riot like this, built entirely around liberation of territory from the cops, that the complete absence of this protagonist, while seemingly realising the riot's very aim, deprives it of the dynamic which enables it to develop. The police, in this sense, are not an external force of order applied by the state to an already rioting mass, but an integral part of the riot" (Endnotes 8. August 2011).

\section{Riot als Protestform der Krise}

Betrachtet man die Ursachen, die als Gründe für Riots in den frühindustrialisierten Ländern ausgemacht werden, eröffnet sich eine fragwürdige Dichotomie. Während bei den Riots in London, Paris oder Ferguson materielle Gründe und somit soziale Ungleichheit durchaus als Ursache anerkannt werden, spricht man den marginalisierten und/oder rassifizierten Jugendlichen politisch bewusstes Handeln ab. Hingegen werden bei Riots, die durch Linksradikale oder AnarchistInnen zustande kommen, keine soziostrukturellen Gründe gesehen, sondern eine fehlgeleitete, gar terroristische Ideologie als Ursache ausgemacht. So ergibt sich das Bild, dass die einen unter jenen gesellschaftlichen Verhältnissen leiden, die die anderen vornehmlich analysieren. Während also die geteilte Identität der marginalisierten Vorstadtjugendlichen eher auf einer geteilten Erfahrung der Ausgrenzung beruht, ist es bei den aus der Mittelschicht kommenden und meist akademischen Linksradikalen vor allem die geteilte politische Haltung, die zur kollektiven Identität beiträgt. Doch diese vereinfachte Zuspitzung entspricht nicht der Realität. Aufgrund der sozioökonomischen Entwicklungen und Ausschlüssen verbinden sich zunehmend die Ebenen von Erfahrung und Erkenntnis. Auch bei Kindern der Mittelschicht lassen sich Frust, soziale Unsicherheit und Angst beobachten. Aufgrund der herrschenden Konkurrenzlogik macht sich zunehmend ein Gefühl breit, überflüssig oder austauschbar zu sein. Wenn man nicht die nötigen beruflichen und sozialen Kompetenzen aufzuweisen hat, kann man sich auf dem Arbeitsmarkt nicht gegen andere durchsetzen. Der soziale Abstieg wird somit zur steten Bedrohung. Darüber hinaus ist den Ausschreitungen hier wie da gemeinsam, dass sie überwiegend von männlichen Akteuren ausgeübt werden. Versteht man die derzeiti- 
gen Riots als einen Ausdruck einer gesellschaftlichen Krise, in der die soziale und politische Integration von Teilen der Gesellschaft droht fragil zu werden, so wird deutlich, dass vom ökonomischen und sozialen Statusverlust vor allem Männer betroffen sind. ${ }^{28}$

Die sozial marginalisierten und linksradikalen RandaliererInnen teilen somit das Gefühl der Handlungsohnmacht und eine pessimistische Zukunftserwartung in Bezug auf ihre individuellen Lebensperspektiven, mitunter auch hinsichtlich der ökologischen und politischen Entwicklung der Welt. Die Mehrheit der randalierenden AktivistInnen und erlebnishungrigen, szenefremden Jugendlichen eint daher weniger eine geteilte sozioökonomische Position oder ein politisch-kultureller Konsens, sie verfügen auch über kein gemeinsames politisches Programm. Die Ausschreitungen offenbaren vielmehr das Gegenteil: „The riot seeks to preserve nothing, to affirm nothing but perhaps a shared antagonist, a shared misery, a shared negation" (Clover 2016: 150). Obwohl Clovers Analysen primär in Bezug auf den angloamerikanischen Kontext entstanden sind und keinen globalen Deutungsanspruch erheben, lohnt sich meines Erachtens eine Adaption auf hiesige Verhältnisse, da die grundlegenden politökonomischen Entwicklungen ähnliche sind. So bestätigen die sich in den letzten Jahren transformierenden Protestartikulationen in den früh industrialisierten Ländern Clovers These über den Zusammenhang von makroökonomischen Entwicklungen und sozialen Konflikten. Nicht nur die Riots in den armen Vierteln und Vororten, sondern auch die Occupy-Bewegung und das Protestrepertoire während des G20-Gipfels lesen sich wie eine Bestätigung der Annahme Clovers, der für die nächsten Jahre vor allem Blockaden, Plünderungen, Barrikaden, Besetzungen und Riots kommen sieht, also Proteste, die vor allem die Zirkulation von Gütern und Waren beeinträchtigen.

Auch wenn Riots mit Gewalt, Sprachlosigkeit und fehlenden Forderungen einhergehen, kann man sie angesichts der sozialstrukturellen und ideellen Gründe durchaus - wie Balibar es ausdrückt - als einen Akt zur Erlangung von Repräsentation, Anerkennung und Partizipation deuten (Balibar 2007: 58). Sie stellen damit eine, wenn auch strafrechtlich relevante, politische Pro-

28 Zwar trifft die derzeit steigende soziale Ungleichheit auch Frauen, aber prekäre und schlecht bezahlte Arbeitsplätze gehören seit Jahrzehnten zur Lebensrealität vieler Frauen. testhandlung dar. Dementsprechend sind sowohl die Straßenschlachten in Hamburg als auch die Größe der überwiegend friedlichen G20-Proteste ein Ausdruck einer gesellschaftlichen Krise. Doch nur mit politökonomischen und daran geknüpften ideellen Argumenten kann der Ausbruch eines Riots nicht hinreichend erklärt werden. So lassen sich Riots außerdem als affektiver Prozess begreifen, mit dem die Alltäglichkeit des Lebens unterbrochen wird und der für die Teilnehmenden offenbar eine Befriedigung bringt, die sie sonst nicht zu finden wissen. Aber nicht nur die Affekte, sondern auch die situative Zuspitzung des Konfliktes zwischen Polizei und ihren GegnerInnen bedingen Form und Ausbruch der Riots. Die Polizei ist Teil der Ausschreitungen, da sie als Katalysator und als Gegenüber dient, wodurch sich die heterogenen AkteurInnen zu einem flüchtigen Kollektiv zusammenfinden, mit dem sie sich den urbanen Raum zeitweise anzueignen und ihn zu beherrschen versuchen. Die gegenwärtigen gesellschaftlichen Konflikte und die soziale Polarisierung entladen sich gleichsam in der Auseinandersetzung mit der Polizei.

Vor dem Hintergrund des konstant niedrigen Wirtschaftswachstums und einer Umstrukturierung der Arbeitswelt, die mit zunehmender ökonomischer Ungleichheit und Marginalisierung von Teilen der Bevölkerung einhergeht, stellt sich somit die Frage, ob Protest weiterhin über herkömmliche Mechanismen der Repräsentation aufgefangen werden kann. Diversifizierung des Arbeitsmarkts und die zunehmende Prekarisierung haben die Anrufung der traditionellen Arbeiterklasse als das politische Subjekt schon lange obsolet gemacht. In den neu entstandenen sozialen Bewegungen seit den 196oer-Jahren war soziale Sicherheit als Leitgedanke sekundär, vielmehr boten sie ein Ventil für ein kulturelles Unbehagen, den Frust über „Fremdbestimmung“ und fehlende ,individuelle Autonomie“ (Dörre 2012: 53). Zwar haben sich in den vergangenen Jahren die sozialen Kämpfe vervielfältigt und lassen sich in unterschiedlichen gesellschaftlichen Bereichen finden. Doch solange die hiesigen gesellschaftlichen Verhältnisse in ihrer aktuellen Konfiguration verbleiben und eine Antwort auf die neue soziale Frage (Castel 2008) weiterhin aussteht, wird es immer wieder zu gewaltvollen Ausschreitungen kommen, wie sie sich in Hamburg während des G2o-Gipfels ereignet haben. 


\section{Literatur}

afp (15. Juli 2017): Frankreich: Knapp 900 Autos bei FeiertagsRandale in Frankreich angezündet. Online: https://www. faz.net/aktuell/gesellschaft/kriminalitaet/randale-amfeiertag-knapp-90o-autos-in-frankreich-angezuendet-15108295.html [28.12.2018].

Altenried, M. (2012): Aufstände, Rassismus und die Krise des Kapitalismus: England im Ausnahmezustand. Münster: edition assemblage.

Aminzade, R./McAdam, D. (2001): Emotions and Contentious Politics. In: Aminzade, R./Goldstone, J. A./McAdam, D./Perry, E. J./Sewell, W. H. Jr/Tarrow, S./Tilley, Ch. (Hg.): Silence and Voice in the Study of Contentious Politics. Cambridge: Cambridge University Press, 14-50.

Anonym (2017): Da war doch was in Hamburg. magazin. Online: http://www.magazinredaktion.tk/docs/hh.pdf [14.5.2018].

Auyero, J. (2001): Glocal Riots. International Sociology, 16 (1), $33-53$.

Balibar, É. (2007): Uprisings in the Banlieues. Constellations, $14(1), 47-71$.

Bareis, E./Bojadzijev, M. (2011): Jenseits von Forderungen und Organisierung: Revolten in den französischen Vorstädten. Das Argument 52, (6), 839-848.

BlockG2O (2. April 2017): Aufruf: Colour the red zone! Online: https://www.g2ohamburg.org/de/content/hamburg7-juli-2017-blockg2O-colour-red-zone [24.08.2018].

$\mathrm{BpB}$, Bundeszentrale für politische Bildung (2010): Erwerbstätigkeit von Frauen. Online: www.bpb.de/system/files/ pdf/O4NQPA.pdf [5.5.2018].

$\mathrm{BpB}$, Bundeszentrale für politische Bildung (31. Januar 2014): Das Normalarbeitsverhältnis. Online: https://www.bpb. de/politik/innenpolitik/arbeitsmarktpolitik/178192/ normalarbeitsverhaeltnis? $\mathrm{p}=$ all [29.8.2017] .

Calhoun, C. (2001): Putting emotions in their place. In: Goodwin. J./Jasper, J. M./Polletta, F. (Hg.): Passionate Politics: Emotions and Social Movements. Chicago: University Of Chicago Press, 45-57.

Carini, M. (6. Juni 2012): Proteste gegen Sozial-Kahlschlag: Auftakt zu einem heißen Herbst. Die Tageszeitung: taz. Online: http://www.taz.de/!5092143/ [30.8.2017].

Castel, R. (2008): Die Metamorphosen der sozialen Frage: eine Chronik der Lohnarbeit. 2. Auflage, Konstanz: UVK.

Clover, J. (2016): Riot. Strike. Riot.: The new era of uprisings. London: Verso.

Collins, R. (2008): Violence: a micro-sociological theory. Princeton: Princeton University Press.

Comité Invisible (Hg.) (2010): Der kommende Aufstand. 5. Auflage, Hamburg: Edition Nautilus.

CrimethInc. (2010): Say You Want an Insurrection. CrimethInc. Online: https://crimethinc.com/2010/01/o7/sayyou-want-an-insurrection [14.8.2017].

CrimethInc. Ex-Workers'Collective (2018): Der G2o-Gipfel 2017 in Hamburg - umfassender Bericht und Analyse.
In: Dellwo, K.-H./Weiler, P./Szepanski, A. (Hg.): Riot. Was war da los in Hamburg? Theorie und Praxis der kollektiven Aktion. Hamburg: Laika, 17-56.

Della Porta, D. (2006): Social movements, political violence, and the state: a comparative analysis of Italy and Germany. Cambridge: Cambridge University Press.

Della Porta, D./Gbikpi, B. (2012): The Riots: A Dynamic View. In: Seferiades, S./Johnston, H. (Hg.): Violent Protest, Contentious Politics, and the Neoliberal State. Farnham/ Burlington: Ashgate, 87-102.

Der Paritätische Gesamtverband (2017): Menschenwürde ist Menschenrecht. Bericht zur Armutsentwicklung in Deutschland. Online: https://www.isl-ev.de/attachments/ article/1726/armutsbericht-2017_aktuell.pdf [10.5.2018].

Doell, D. (2018): Wake-up Call - Der Mikro-Aufstand von Hamburg als materielles und diskursives Ereignis. In: Dellwo, K.-H./Szepanski, A. (Hg.): Riot. Was war da los in Hamburg? Theorie und Praxis der kollektiven Aktion. Hamburg: Laika, 77-90.

Dörre, K. (2012): Die neue Landnahme. Dynamiken und Grenzen des Finanzmarktkapitalismus. In: Lessenich, S./Dörre, K./Rosa, H. (Hg.): Soziologie - Kapitalismus - Kritik: eine Debatte. Frankfurt am Main: Suhrkamp, 21-86.

Dörre, K. (2015): Génération Précaire - ein europäisches Phänomen. In: Busch, M./Jeskow, J./Stutz, R. (Hg.): Zwischen Prekarisierung und Protest: Die Lebenslagen und Generationsbilder von Jugendlichen in Ost und West. Bielefeld: Transcript, 39-74.

dpa (7. September 2017): G20-Gipfel: 50 Prozent mehr Polizisten im Einsatz als bekannt. Spiegel Online. Online: http://www.spiegel.de/politik/deutschland/g2O-gipfel50-prozent-mehr-polizisten-im-einsatz-als-bisher-bekannt-a-1166651.html [24.08.2018].

Dragonowl, L. (2015): Against Identity Politics. The Anarchist Library. Online: https://theanarchistlibrary.org/library/ lupus-dragonowl-against-identity-politics [6.9.2017].

Dzudzek, I./Müller, M. (2013): Der Lärm des Politischen. Die Londoner riots 2011 und ihre politischen Subjekte. sublurban. Zeitschrift für kritische Stadtforschung, 2 (1), $17-40$.

Eder, S./Kasumov, A. (8. Juli 2017): Krawall-Nacht in Hamburg: Wie die Schanze im Chaos versank. FAZ.NET. Online: abrufbar unter: https://www.faz.net/aktuell/g20-gipfel/gewalt-bei-g2o-rechtsfreie-zone-schanzenviertel-15096937.html [24.8.2018].

Ehrenberg, A. (2008): Das erschöpfte Selbst: Depression und Gesellschaft in der Gegenwart. 7. Auflage, Frankfurt am Main: Campus.

Endnotes (8. August 2011): A Rising Tide Lifts All Boats. Crisis era struggles in Britain. Online: https://endnotes.org. $\mathrm{uk} /$ issues/3/en/endnotes-a-rising-tide-lifts-all-boats [23.8.2018].

Fisher, M. (2015): Gespenster meines Lebens: Depression, Hauntology und die verlorene Zukunft. Berlin: Edition Tiamat. 
Fox Piven, F. (2012): Protest movements and violence. In: Seferiades, S./Johnston, H. (Hg.): Violent Protest, Contentious Politics, and the Neoliberal State. Farnham/Burlington: Routledge, 19-28.

Frenzel, F./Knopp, Ph./Ullrich, P. (2018): Eine lange Woche im Juli. Chronologie der Konflikte um den G2o-Gipfel in Hamburg (2.-9. Juli 2017). Online: https://g20.protestinstitut.eu/chronologie/ [31.10.2018].

Frenzel, J./Greif, Ph./Klein, F./Uhlmann, S. (2016): Riots Zur Verortung eines unscharfen Phänomens. sub\urban. Zeitschrift für kritische Stadtforschung, 4 (1), 7-24.

Gelderloos, P. (2013): Aufstand vs. Organisation. Betrachtungen aus Griechenland über eine sinnlose Spaltung. Online: https://translationcollective.files.wordpress. com/2013/o3/aufstand-vs-organisation.pdf [24.08.2018].

Goodwin, J./Jasper, J. M./Polletta, F. (2004): Emotional Dimensions of Social Movements. In: Snow, D. A./Soule, S. A./Kriesi, H. (Hg.): The Blackwell Companion to Social Movements. Maldon/ Oxford/ Victoria: Wiley, 413-432.

Granvista Media (2018): Vor dem Knall. Online: https://www. g2ofilm.de/ [15.09.2018].

Greif, Philippe (2012): London Calling Paris. Zur Relevanz der Kategorie Männlichkeit für eine intersektionale Analyseperspektive auf urbane Jugendaufstände in Europa. gender...politik...online. Online: https://www. fu-berlin.de/sites/gpo/soz_eth/Frauen-_M__nnerforschung/London_Calling_Paris__Zur_Relevanz_der_ Kategorie_M_nnlichkeit_f__r_eine_intersektionale_ Analyseperspektive_auf_urbane_Jugendaufst_nde_in_ Europa/Greif-komplett-Nov12.pdf [24.08.2018].

Hall, S. (1994): Rassismus und kulturelle Identität. Hamburg: Argument Verlag.

Hamburg, Hamburger Abendblatt (19. Juli 2017): Polizei: „Trittbrettfahrer" für Krawalle mitverantwortlich. Online: https://www.abendblatt.de/hamburg/kommunales/article211301463/Andy-Grote-bezieht-im-InnenausschussStellung-zu-G2o.html [25.1.2018].

Harvey, D. (2012): Rebellische Städte. Berlin: Suhrkamp.

Imaginäre Partei (2018): Unsere Nächte brauchen zum Leuchten keine Sterne mehr. In: Lotzer, S. (Hg.): Winter is coming. Wien/Paris/Hamburg: bahoe books, 116-130.

Institut für Protest- und Bewegungsforschung (November 2017): Ergebnisse der Befragung von Demonstrierenden und der Beobachtung des Polizeieinsatzes. Online: https:// protestinstitut.eu/wp-content/uploads/2017/11/NoG2O_ ipb-working-paper.pdf [20.8.2018].

James, M. (2011): Behind the riots: what young people think about the 2011 summer unrest. The Guardian. Online: https://www.theguardian.com/society/2011/sep/05/youngpeople-2011-summer-unrest [25.8.2018].

Jasper, J. M. (2011): Emotions and Social Movements: Twenty Years of Theory and Research. Annual Review of Sociology, 37, 285-303.

Jasper, J. M./Thompson, A.K. (2016): Did Someone Say Riot? James M. Jasper in Conversation with AK Thompson. Social Movement Studies, 15, 216-230.
Jürgens, K. (Dezember 2010): Deutschland in der Reproduktionskrise. Leviathan, 38, 559-587.

Jürgens, K. (2012): Arbeit und Reproduktion. In: Dörre, K./ Sauer, D./Wittke, V. (Hg.): Kapitalismustheorie und Arbeit: neue Ansätze soziologischer Kritik. Frankfurt am Main: Campus, 273-288.

Komitee für Grundrechte und Demokratie e.V. (2017): Geschichte der Eskalation eines einwöchigen Protestgeschehens. Demonstrationsbeobachtung des Komitees für Grundrechte und Demokratie vom 2. bis 8. Juli 2017 in Hamburg zum G2O. Online: http://www.grundrechtekomitee.de/sites/default/files/G2O_Protest.pdf [1.9.2017].

Landesamt für Verfassungsschutz (2017): Verfassungsschutzbericht 2016. Online: https://www.hamburg.de/contentb lob/8873924/38b7f14ba1da5dd3693b6b1a833d9c43/data/ verfassungsschutzbericht-2016-lfv-hh.pdf [3.9.2018].

Loick, D. (28. Juli 2017): Polizei als Risiko für Demokratie. Frankfurter Rundschau. Online: http://www.fr.de/kultur/gewaltmonopol-die-polizei-als-risiko-fuer-die-demokratie-a-1321343 [24.8.2018].

Lotzer, S. (2018a): Schanzenblues. In: Dellwo, K.-H./Weiler, P./Szepanski, A. (Hg.): Riot. Was war da los in Hamburg? Theorie und Praxis der kollektiven Aktion. Hamburg: Laika, 91-96.

Lotzer, S. (2018b): Winter is coming. In: Lotzer, S. (Hg.): Winter is coming. Wien/Paris/Hamburg: bahoe books, 131-134.

Martinez, E. (B.) (2004): Where Was the Color in Seattle? Looking for Reasons Why the Great Battle was So White. Monthly Review, 52 (3), 141.

Meister, M./Niebuhr, A./Stöckmann, A. (2017): Die Arbeitsmarktsituation von Migrantinnen und Migranten in Hamburg. Online: http://doku.iab.de/regional/N/2017/ regional_n_0117.pdf [1.9.2017].

Melchers, C. (20. Juli 2017): Willkommen im Wahnsinn. jungle.world. Online: https://jungle.world/artikel/2017/29/ willkommen-im-wahnsinn [3.9.2017].

Merkel, W./Petring, A. (2011): Partizipation und Inklusion. Ein Report der Friedrich-Ebert-Stiftung. Online: http:// library.fes.de/pdf-files/akademie/o8036.pdf [3.8.2017].

Mester, V. (24. September 2014): Aktuelle Nachrichten - Hamburger Abendblatt. Online: abrufbar unter: https://www. abendblatt.de/wirtschaft/article132561648/42-0oo-Millionaere-und-18-Milliardaere-leben-in-Hamburg.html [23.8.2017].

Mücke, J./Rinn, M. (2016): Keine riots in Deutschland? Die Ereignisse in Hamburg-Altona im Sommer 2013. sub $\backslash$ urban. Zeitschrift für kritische Stadtforschung, 4 (1), 111-130.

Mullis, D. (2018): G2o in Hamburg. sub\urban. Zeitschrift für kritische Stadtforschung, 6 (1), 29-50.

Nachtwey, O. (2016): Die Abstiegsgesellschaft: über das Aufbegehren in der regressiven Moderne. Berlin: Suhrkamp.

Nassehi, A. (13. Juli 2017): G20: Eine Linke braucht es nicht mehr. Die Zeit. Online: http://www.zeit.de/kultur/2017-07/g20-linke-gewalt-kapitalismuskritik-globalisierung-essay [23.8.2017]. 
NDR (11. Juli 2017): Druck auf Rote Flora wächst nach G20. Online: https://www.ndr.de/nachrichten/hamburg/ Druck-auf-Rote-Flora-waechst-nach-G2o,gzwanzig212. html [5.9.2017].

NoG2o International (2017): We are many! We are loud! The world will hear us -. Online: http://g2o-protest.info/weare-many-we-are-loud-\%cf\% 84 he-world-will-hear-us/ [9.5.2018].

Piketty, T. (2016): Das Kapital im 21. Jahrhundert. 8. Auflage, München: C. H. Beck.

Pohl, G./Wicher, K. (2013): Vorwort. In: Pohl, G./Wicher, K. (Hg.): Hamburg: gespaltene Stadt? Soziale Entwicklungen in der Metropole. Hamburg: VSA, 7-12.

Polke-Majewski, K. (8. Juli 2017): G20-Ausschreitungen: Die Schlacht auf der Schanze. Zeit Online. Online: https:// www.zeit.de/gesellschaft/2017-07/g20-ausschreitungenschanze-schwarzer-block-polizei [24.8.2018].

Pritchard, D./Pakes, F. J. (2014): Exploring the Global Stage: Globalisation, Riots, Unrest and Protest. In: Pritchard, D./Pakes, F. J. (Hg.): Riot, unrest and protest on the global stage. London: Palgrave Macmillan, 1-11.

Rauschenbach, K. (24. August 2017): Hamburg war ein Fest. Jungle World. Online: https://jungle.world/index.php/ artikel/2017/34/hamburg-war-ein-fest [30.8.2017].

Reicher, S./Stott, C. (2011): Mad Mobs and Englishmen?: Myths and realities of the 2011 riots. London: Robinson.

Reisin, A. (20. Juli 2017): Polizeiübergriff bei G2o: Ein verhängnisvoller Abend. Online: https://daserste.ndr.de/panorama/aktuell/Polizeiuebergriff-bei-G2o-Ein-verhaengnisvoller-Abend,gzwanzig258.html [21.8.2018].

Rilrec, L. von (2018): Festival der Demokratie. Online: https:// www.festival-der-demokratie.de/de/ [24.08.2018].

Rosa, H. (2011): Beschleunigung und Depression. Überlegungen zum Zeitverhältnis der Moderne. Psyche - Zeitschrift für Psychoanalyse, 65 (11), 1041-1060.

Rose, W./Klages, S. (2011): Privater Reichtum - öffentliche Armut. In: Pohl, G./Wicher, K. (Hg.): Armes Reiches Hamburg: Metropole zwischen Wohlstand und Armut. Hamburg: VSA, 14-27.

Roter Aufbau Hamburg (2015): Selbstverständnis. Online: http://roter-aufbau.de/selbstverstaendnis/ [5.9.2018].

Rucht, D. (2016): Riots - Anmerkungen zu Begriff und Konzept. sublurban. Zeitschrift für kritische Stadtforschung, 4 (1), 25-30.

Sassen, S. (2015): Ausgrenzungen: Brutalität und Komplexität in der globalen Wirtschaft. Frankfurt am Main: S. Fischer.

Seraphim, S./Johnston, H. (2012a): The Dynamics of Violent Protest: Emotions, Repression and Disruptive Deficit. In: Seferiades, S./Johnston, H. (Hg.): Violent Protest, Contentious Politics, and the Neoliberal State. Farnham/ Burlington: Routledge, 3-18.

Seferiades, S./Johnston, H. (2012b): The Greek December. In: Seferiades, S./Johnston, H. (Hg.): Violent Protest, Contentious Politics, and the Neoliberal State. Farnham/Burlington: Routledge, 149-156.
Serrao, M. F. (11. Juli 2017): Nach G2o-Gipfel: Die Gefahren des Linksextremismus. Neue Zürcher Zeitung. Online: https://www.nzz.ch/international/g-2o-gipfel-in-hamburg-brennen-fuer-den-revolutionaeren-umsturzld.1305347 [14.5.2018].

Slater, T. (2011): From ,Criminality' to Marginality: Rioting Against a Broken State. Human Geography. Online: https://hugeog.com/v4n3-criminality-to-marginality [10.3.2016]

St. Pauli Selber Machen (29. Mai 2017): Unser Flyer für den Stadtteil zum G2O-Gipfel. Online: http://www.st-pauliselber-machen.de/?p=24183 [5.9.2017].

Statistische Ämter des Bundes und der Länder (2017): Sozialberichterstattung - Armutsgefährdungsquoten. Online: http://www.amtliche-sozialberichterstattung.de/A1armutsgefaehrdungsquoten.html [7.9.2017].

Statistisches Amt für Hamburg und Schleswig-Holstein (15. Februar 2015): Bürgerschaftswahlen in Hamburg 2015. Online: http://www.statistik-nord.de/fileadmin/maps/ election_2015_hh_bue_e/index.html [5.9.2017].

Thompson, AK (2010): Black Bloc, White Riot: Antiglobalization and the Genealogy of Dissent. Edinburgh/Oakland: AK Press.

Thrasher, Steven W. (19. August 2014): The real looting of Ferguson: its black citizens never had a chance to get by. The Guardian. Online: https://www.theguardian.com/ commentisfree/2014/aug/19/ferguson-looting-nationalguard-black-citizens-get-by [28.8.2018].

Tilly, Ch.(2003): The Politics of Collective Violence. Cambridge: Cambridge University Press.

Tiratelli, M. (2018): Reclaiming the everyday: the situational dynamics of the 2011 London Riots. Social Movement Studies, 17 (1), 64-84.

translationcollective (8. März 2010): Einige Notizen zu aufständischem Anarchismus. translationcollective. Online: https://translationcollective.wordpress.com/2010/03/o8/ einige-notizen-zu-aufstandischem-anarchismus/ [30.8.2017].

Trott, B. (2014): Research and the Riots: Politics and England's 2011 Urban Uprisings. CritCom. Online: http://councilforeuropeanstudies.org/critcom/research-and-theriots-politics-and-englands-2011-urban-uprisings/ [19.3.2016].

UmsGanze (2017): Shut down the logistics of capital. Online: https://g2otohell.blackblogs.org/2017/06/o7/shut-downthe-logistics-of-capital-auch-in-bremen/ [15.5.2018].

Wacquant, L. (2006): Das Janusgesicht des Ghettos und andere Essays. Basel: Birkhäuser.

Waddington, D./King, M. (2012): Contemporary French and British urban riots: an exploration of the underlying political dimensions. In: Seferiades, S./Johnston, H. (Hg.): Violent Protest, Contentious Politics, and the Neoliberal State. Farnham/Burlington: Routledge, 119-132.

ZDF (9. Juli 2017): Pressekonferenz von Polizei und Stadt Hamburg zur G2o-Bilanz. Online: https://www.zdf.de/ uri/oo8e6efb-e876-429c-b9af-8ocbe675573e [11.5.2018]. 